\title{
Immunosurveillance and therapy of multiple myeloma are CD226 dependent
}

\author{
Camille Guillerey, ${ }^{1,2}$ Lucas Ferrari de Andrade, ${ }^{1,3}$ Slavica Vuckovic, ${ }^{4}$ Kim Miles, ${ }^{1}$ Shin Foong Ngiow, ${ }^{1}$ Michelle C.R. Yong, ${ }^{5}$ \\ Michele W.L. Teng, ${ }^{5}$ Marco Colonna, ${ }^{6}$ David S. Ritchie, ${ }^{7}$ Martha Chesi, ${ }^{8}$ P. Leif Bergsagel, ${ }^{8}$ Geoffrey R. Hill, ${ }^{4}$ \\ Mark J. Smyth, ${ }^{1,2}$ and Ludovic Martinet ${ }^{1,9}$ \\ IImmunology of Cancer and Infection Laboratory, QIMR Berghofer Medical Research Institute, Herston, Queensland, Australia. ${ }^{2}$ School of Medicine, University of Queensland, Herston, Queensland, Australia. \\ 3'élulas Inflamatórias e Neoplásicas group, Universidade Federal do Paraná, Curitiba, Paraná, Brazil. "BBone Marrow Transplantation and ${ }^{5}$ Cancer Immunoregulation and Immunotherapy Laboratory, \\ QIMR Berghofer Medical Research Institute, Herston, Queensland, Australia. ${ }^{6}$ Department of Pathology and Immunology, Washington University School of Medicine, St. Louis, Missouri, USA. \\ ${ }^{7}$ Sir Peter MacCallum Department of Oncology, University of Melbourne, Parkville, Victoria, Australia. ${ }^{8}$ Comprehensive Cancer Center, Mayo Clinic, Scottsdale, Arizona, USA. \\ ${ }^{9}$ INSERM UMR 1037, Cancer Research Center of Toulouse, Toulouse, France.
}

\begin{abstract}
Multiple myeloma (MM) is an age-dependent hematological malignancy. Evaluation of immune interactions that drive MM relies on in vitro experiments that do not reflect the complex cellular stroma involved in MM pathogenesis. Here we used $\mathrm{Vk}^{*}$ MYC transgenic mice, which spontaneously develop MM, and demonstrated that the immune system plays a critical role in the control of MM progression and the response to treatment. We monitored Vk*MYC mice that had been crossed with Cd226 mutant mice over a period of 3 years and found that CD226 limits spontaneous MM development. The CD226dependent anti-myeloma immune response against transplanted Vk* MYC MM cells was mediated both by NK and CD8 ${ }^{+}$ T cells through perforin and IFN- $\gamma$ pathways. Moreover, CD226 expression was required for optimal antimyeloma efficacy of cyclophosphamide (CTX) and bortezomib (Btz), which are both standardly used to manage MM in patients. Activation of costimulatory receptor $\mathrm{CD} 137$ with $\mathrm{mAb}$ (4-1BB) exerted strong antimyeloma activity, while inhibition of coinhibitory receptors PD-1 and CTLA-4 had no effect. Taken together, the results of this study provide in vivo evidence that CD226 is important for MM immunosurveillance and indicate that specific immune components should be targeted for optimal MM treatment efficacy. As progressive immunosuppression associates with MM development, strategies aimed to increase immune functions may have important therapeutic implications in MM.
\end{abstract}

\section{Introduction}

Multiple myeloma (MM) is the second most common hematological malignancy in the world (1). Despite recent progress in new therapeutic options, this disease remains incurable, with a median survival not exceeding 5 years in adults. $\mathrm{MM}$ is characterized by the proliferation of clonal, long-lived plasma cells (PCs) within the $\mathrm{BM}$ associated with bone destruction, serum monoclonal gammopathy, and organ dysfunction (1). MM often evolves at a slow rate from an age-dependent premalignant stage called monoclonal gammopathy of undetermined significance (MGUS) (2). The driving forces that promote the evolution from MGUS to active MM have remained obscure until now. Although a number of oncogenic events are associated with MM evolution $(2,3)$, the pathogenesis of $\mathrm{MM}$ could reflect not only cell-intrinsic alterations, but also the impact of host immune reactions. Indeed, accumulating evidence over the past decades demonstrates that the immune system plays a pivotal role in the surveillance of cancer $(4,5)$. Both innate and adaptive immune cells can recognize and destroy malignant PCs

Authorship note: Camille Guillerey, Lucas Ferrari de Andrade, Mark J. Smyth, and Ludovic Martinet contributed equally to this work.

Conflict of interest: The authors have declared that no conflict of interest exists. Submitted: May 21, 2014; Accepted: March 12, 2015.

Reference information: J Clin Invest. 2015;125(5):2077-2089. doi:10.1172/JCI77181.
(6-9), and progressive immune suppression has been involved in the progression of MM $(10,11)$. Additional proof of MM control by immune components derives from the clinical use of immunomodulatory drugs (IMiDs) whose antimyeloma properties rely, at least partially, on the costimulation of T and NK cells (12-14).

The interaction between CD226 (DNAM-1) with its ligands CD155 (PVR, necl-5) and CD112 (nectin 2) has recently emerged as a critical mechanism of immune defence against a wide range of malignancies (15-18). CD226 was originally described as an adhesion coreceptor stimulating NK cell- and $\mathrm{CD}^{+} \mathrm{T}$ cell-mediated cytotoxicity against tumor cells (19). It has now become clear that this receptor has broader implications than originally thought in controlling NK cell and T cell function $(20,21)$. CD226 has been implicated in immunological synapse formation (22), T cell proliferation and differentiation (23), and cytokine secretion $(21,24)$. Interestingly, CD112 and CD155 are often present at the surface of malignant PCs in MM patients, and CD226 strongly contributes to NK cell-mediated killing of MM cells in vitro $(8,9)$. Moreover, therapeutic agents commonly used in the management of MM, such as melphalan and bortezomib (Btz), upregulate CD226 ligands and sensitize MM cells to NK cell-mediated cytotoxicity (25, 26). Altogether, this suggests that CD226 may play an important role in MM pathogenesis, and modulation of this pathway may have therapeutic implications. 
Most of our understanding of immune interactions with MM relies on in vitro experiments. Given the importance of BM stroma in the biology of MM, and the complex cellular and cytokine interactions contributing to $\mathrm{MM}$ development, the previous results should be taken with some caution (27). Vk* MYC transgenic mice, which drive sporadic MYC oncogene expression in germinal center $\mathrm{B}$ cells, have recently emerged as the most relevant mouse model of MM currently available $(28,29)$. $\mathrm{Vk}^{*} \mathrm{MYC}$ mice spontaneously develop MGUS that progresses into indolent MM over 20-50 weeks, and closely mirrors human MM pathological progression. This includes PC neoplasia restricted to the $\mathrm{BM}$, bone destruction, and monoclonal gammopathy (28). $\mathrm{Vk}^{*} \mathrm{MYC}$ mice were shown to respond to most of the conventional antimyeloma drugs and thus represent a valuable preclinical tool to test new MM therapies in immune-competent hosts $(30,31)$.

In this study using this unique mouse model of MM, we demonstrate the importance of $\mathrm{NK}$ and $\mathrm{CD}^{+} \mathrm{T}$ cells in $\mathrm{MM}$ immunosurveillance and response to treatment in vivo through CD226 and CD155 interactions.

\section{Results}

CD226 limits spontaneous MM burden in $V k^{*} M Y C$ mice. To assess the role of CD226 in MM emergence and progression, we crossed $\mathrm{Vk}^{*} \mathrm{MYC}$ with $\mathrm{Cd} 226^{--}$mice. The presence of monoclonal Ig (M-spike) and the level of $\gamma$-globulin in the serum were quantified in $\mathrm{Cd} 226^{-/}, \mathrm{Cd} 226^{+/-}$, and $\mathrm{Cd} 226^{+/+} \mathrm{Vk}^{*} \mathrm{MYC}$ transgenic and nontransgenic littermates by serum protein electrophoresis (SPEP). $C d 226^{+/+}$, $\mathrm{Cd} 226^{-/}$, and $\mathrm{Cd} 226^{+/-} \mathrm{Vk}^{*} \mathrm{MYC}$ transgenic mice all developed detectable serum paraproteinemia (M-spike) between 200 and 400 days of age (Supplemental Figure 1; supplemental material available online with this article; doi:10.1172/JCI77181DS1) that gradually increased overtime (Figure 1A). As previously described (28), some aged nontransgenic littermates also developed mild gammopathy irrespective of $C d 226$ genotype (Supplemental Figure 1). Interestingly, although the incidence of M-spike was similar between groups of $\mathrm{Vk}^{*} \mathrm{MYC}$ transgenic mice, we observed that the levels of serum $\gamma$-globulin increased significantly faster in $\mathrm{Cd} 226^{-/-} \mathrm{Vk}^{*} \mathrm{MYC}$ mice than in $\mathrm{Cd} 226^{+/-} \mathrm{Vk}^{*} \mathrm{MYC}$ and $\mathrm{Cd} 226^{+/+} \mathrm{Vk}^{*} \mathrm{MYC}$ mice (Figure $1, \mathrm{~A}$ and $\mathrm{B})$. In accordance with their higher serum paraprotein levels, $C d 226^{-/-} \mathrm{Vk}^{*} \mathrm{MYC}$ mice had a significantly reduced overall survival, as compared to $\mathrm{Cd} 226^{+/-}$and WT $\mathrm{Vk}^{*} \mathrm{MYC}$ littermates (Figure 1C and Supplemental Figure 1). By contrast, nontransgenic littermates survived longer than $\mathrm{Vk}^{*} \mathrm{MYC}$ transgenic mice, and they died of "old age" with similar kinetics regardless of the presence or absence of CD226 (Figure 1C and Supplemental Figure 1).

Our results suggested that CD226 does not drastically affect activation induced deaminase-dependent (AID-dependent) spontaneous MYC activation associated with MM emergence in vivo (28, 29). By contrast, it demonstrated that CD226 significantly limited MM progression and improved survival. To confirm this hypothesis, we analyzed the level of PCs in the BM of a second cohort of $C d 226^{-1-}$ and $C d 226^{+/+} \mathrm{Vk}^{\star} \mathrm{MYC}$ transgenic and nontransgenic littermates at an early stage of the disease. We found that $C d 226^{-/-} \mathrm{Vk}^{*} \mathrm{MYC}$ mice had significantly higher percentages and numbers of BM B220 CD138 ${ }^{+}$PCs compared with the WT counterparts (Figure 1, D and E). By contrast, the percentages and numbers of PCs in the BM of both $C d 226^{--}$and WT nontransgenic mice were very low (Figure 1,
D and E). As shown previously (28), malignant PC expansion was mainly restricted to the $\mathrm{BM}$ in $\mathrm{Vk}^{*} \mathrm{MYC}$ mice (Figure 1, D and E, and Supplemental Figure 1). CD155 is a major ligand for CD226 and has been shown to play an important role in the immune recognition of cancer (20). Therefore, we analyzed CD155 expression on B220$\mathrm{CD} 138^{+} \mathrm{PCs}$ naturally arising from WT and $\mathrm{Cd} 226^{-/} \mathrm{Vk}^{*} \mathrm{MYC}$ mice. We found that CD155 was highly expressed on the cell surface of all the malignant PCs developing in transgenic $\mathrm{Vk}^{*} \mathrm{MYC}$ mice (Figure $1 \mathrm{E}$ and Supplemental Figure 2). By contrast, this receptor was only expressed on a small fraction of PCs from normal mice (Supplemental Figure 1). Previous studies suggested that CD226 contributes to shape tumor immunogenicity, a process called cancer immunoediting (4), and that a decrease in CD155 expression on tumor cells may contribute to tumor immune escape (18). However, we observed that the level of $\mathrm{CD} 155$ expression on $\mathrm{B} 220^{-} \mathrm{CD} 138^{+}$malignant PCs was similar between WT and $C d 226^{-/-} \mathrm{Vk}^{*} \mathrm{MYC}$ mice, demonstrating that although CD226 impacts tumor development and survival, there is no detectable immunoediting of malignant PCs through CD226/CD155 interactions (Figure 1F).

CD226 interaction with CD155 on PCs limits MM development. We next investigated the mechanisms involved in the control of MM through the CD226 receptor. Given the timeframes required to modify immune components in the spontaneous $\mathrm{Vk}^{*} \mathrm{MYC}$ model, we decided to use a tractable transplantable model of $\mathrm{Vk}^{*} \mathrm{MYC} \mathrm{MM}$ (Supplemental Figure 2). First, to confirm the role of CD226 receptor in the control of MM growth, WT and $C d 226^{-/-}$mice were injected with $2 \times 10^{6} \mathrm{Vk} 12653 \mathrm{MM}$ cells and were bled every 2 weeks to analyze M-spike levels. We observed a striking increase in serum paraprotein levels between $C d 226^{-/}$and WT mice 3 weeks after injection (Figure 2A). After 5 weeks, the number and the percentage of B220-CD $138^{+}$PCs recovered from the spleen and the BM were both significantly increased in $C d 226^{-/-}$mice as compared to WT mice (Figure 2, B and C, and Supplemental Figure 3). Similar results were obtained with an independent MM cell line derived from $\mathrm{Vk}^{*} \mathrm{MYC}$ transgenic mice, Vk12598 (Figure 2D and Supplemental Figure 2). Finally, we found that mice injected with Vk12598 cells succumbed significantly faster to $\mathrm{MM}$ in the absence of CD226 (Figure 2E). Decreased survival of $C d 226^{-/}$mice compared to WT mice was also observed following the injection of low numbers of Vk12653 cells (Supplemental Figure 3). Given the high level of CD155 expression on $\mathrm{Vk}^{*} \mathrm{MYC}$ cells, we wanted to determine whether CD155 was involved in the CD226 control of $\mathrm{Vk}^{*} \mathrm{MYC}$ MM cells. To this end, we transplanted $\mathrm{Vk}^{*} \mathrm{MYC}$ cells into WT mice injected with anti-CD155, anti-CD226 or control Ig (cIg) as described in Methods. Both anti$\mathrm{CD} 155 \mathrm{mAb}$ - and anti-CD226 mAb-treated mice had higher $\gamma$-globulin levels 3 weeks after injection as compared to cIg-treated mice (Figure 2F). The percentages of $\mathrm{B} 22 \mathrm{O}^{-} \mathrm{CD} 138^{+} \mathrm{PCs}$ in the spleen and the BM were also significantly increased when CD155 or CD226 receptors were blocked (Figure 2G). CD155 can be expressed on several immune cell types after activation, including DC, macrophages, and T lymphocytes. To determine whether endogenous or tumor-derived CD155 was important for the control of MM progression through CD226, we monitored MM growth in $C d 155^{-/}, \mathrm{Cd} 155^{+/-}$, and $\mathrm{Cd} 155^{+/+}$littermates treated with cIg or anti-CD155. We did not observe any significant differences in MM growth between $\mathrm{Cd} 155^{-/}$, $C d 155^{+/-}$, and $C d 155^{+/+}$mice (Figure $2 \mathrm{H}$ ). By contrast, anti-CD155 blockade increased tumor burden in $\mathrm{Cd} 155^{--}$mice, suggesting that 
A

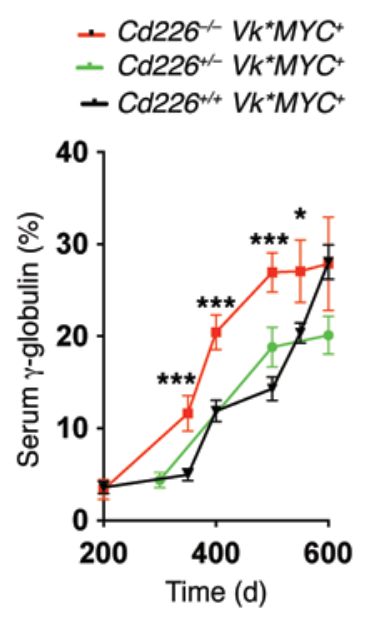

D
Cd226 $6^{+/+}$
O Cd226-
$\Delta \mathrm{Cd}_{226^{*+}} \mathrm{Vk}^{*} \mathrm{MYC}^{+}$
$\triangle C d 226^{-} V k^{*} M Y C^{+}$

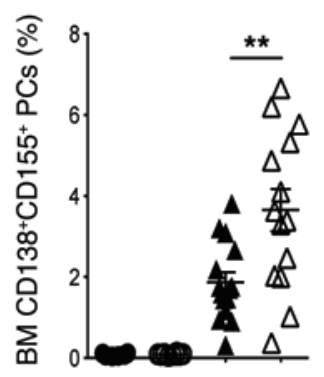

B

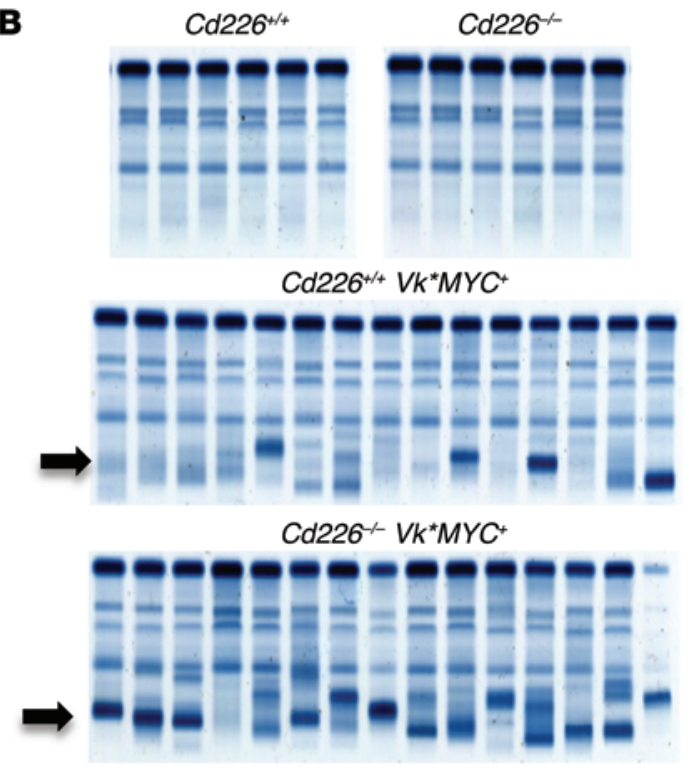

E
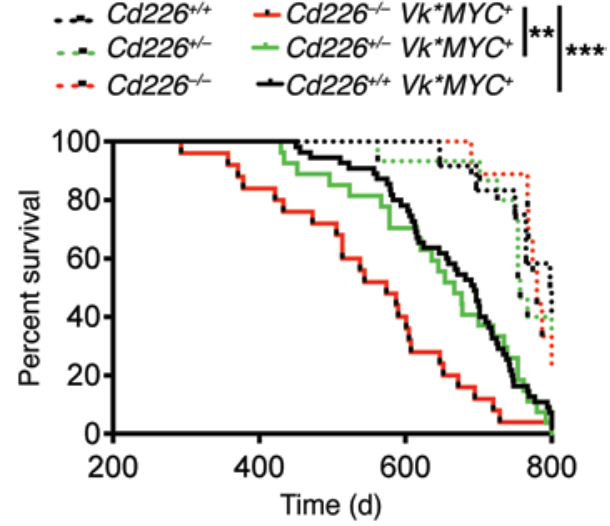

$\mathbf{F}$

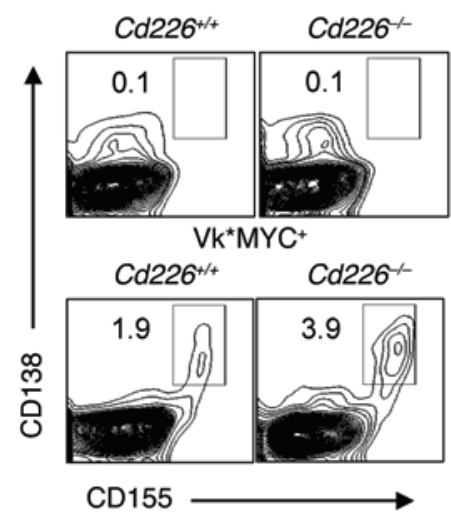

$\Delta \mathrm{Cd226^{*+4 }} \mathrm{Vk}^{*} M Y C^{+}$ $\triangle C d 226^{\sim}-k^{*} M Y C^{+}$

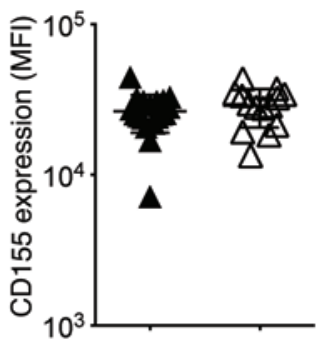

Figure 1. CD226 limits spontaneous MM progression. (A-C) C57BL/6 Cd226 $6^{+/+}(n=55), C d 226^{-/+}(n=27)$, and Cd226 $6^{-/-}(n=25) \mathrm{Vk}^{*} \mathrm{MYC}$ transgenic mice, along with $\mathrm{Cd} 226^{+/+}(n=12), C d 226^{-/+}(n=15)$, and $C d 226^{-/-}(n=9)$ nontransgenic littermate controls, were monitored for MM development (A and $\left.\mathbf{B}\right)$ and survival (C). (A and B) Cd226 $6^{--} \mathrm{Vk}^{*} \mathrm{MYC}$ mice have higher $\gamma$-globulin levels. (A) Graph showing the mean $\gamma$-globulin levels \pm SEM in the serum of the indicated strains of mice over time. (B) Representative SPEP of $C d 226^{+/+}$and $C d 226^{-/-}$Vk* MYC transgenic mice after 500 days. Arrows indicate M-spike. (C) Kaplan Meyer curves showing a significant decrease in the survival of $C d 226^{-1-} \mathrm{Vk}^{*} \mathrm{MYC}$ mice as compared to WT and $C d 226^{-/+} \mathrm{Vk}^{*} \mathrm{MYC}$ littermates. (D-F) $C d 226^{-1-} \mathrm{Vk}^{*} \mathrm{MYC}$ transgenic mice have an increased frequency of malignant PCs expressing CD155 in the BM. The percentage and the number of malignant PCs and their level of CD155 expression were analyzed by FACS in the BM of $C d 226^{+/+}(n=15)$ and $C d 226^{-/-}(n=14) \mathrm{Vk}^{*} \mathrm{MYC}$ transgenic mice and in $C d 226^{+/+}$ $(n=6)$ and $C d 226^{-1-}(n=10)$ nontransgenic littermate controls. ( $\mathbf{D}$ and $\left.\mathbf{E}\right)$ Graphs $(\mathbf{D})$ and representative FACS plots $(\mathbf{E})$ showing the mean frequency and number \pm SEM of CD138+CD155+ malignant PCs in the BM of the indicated strains of mice at 450 days of age. (F) Graph showing similar level of CD155 expression on B220-CD138 BM PCs from $C d 226^{-/-} V^{*} M Y C$ and $C d 226^{+/+} \mathrm{Vk}^{*} \mathrm{MYC}$ mice. Each symbol represents 1 individual mouse. ${ }^{*} P<0.05,{ }^{* *} P<0.01$, ${ }^{* * *} P<0.001$; Mann-Whitney $U$ test (A and $\left.\mathbf{D}\right)$ Mantel-Cox test (C).

tumor CD155 is more important than endogenous CD155 in controlling MM progression. Altogether, these results demonstrate a key role for CD226 interaction with CD155 expressed by PCs in the control of MM development.

$N K$ cells and $C D 8^{+} T$ cells exert antimyeloma activity through perforin and IFN- $\gamma$ pathways. To characterize the immune cells involved in the CD226-dependent antimyeloma response in vivo, we first monitored MM burden in mice depleted of NK cells and/or $\mathrm{CD} 8^{+} \mathrm{T}$ cells using mAbs. Three weeks after injection of Vk12653 cells, both NK cell-depleted mice (anti-asialoGM1 [anti-AsGM1]) and $\mathrm{CD}^{+} \mathrm{T}$ cell-depleted mice (anti-CD8 $\beta$ ) had higher paraprotein levels than cIg-treated mice (Figure 3A and Supplemental

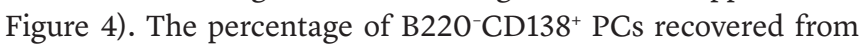

the spleen and the $\mathrm{BM}$ of $\mathrm{NK}$ cell- or $\mathrm{CD} 8^{+} \mathrm{T}$ cell-depleted mice were also significantly increased, as compared with Ig-treated mice (Figure 3A). Interestingly, mice that lacked both NK cells and $\mathrm{CD}^{+} \mathrm{T}$ cells had significantly higher $\gamma$-globulin levels and spleen weight than mice that lacked only one of these two lymphocyte subsets (Figure 3A and Supplemental Figure 4) suggesting that NK cells and CD8 $\mathrm{T}$ cells play additive roles in MM control in vivo.

The granule exocytosis pathway involving perforin and granzymes, and the release of proinflammatory cytokines such as IFN- $\gamma$, are the two main mechanisms contributing to NK cell- and $\mathrm{CD}^{+} \mathrm{T}$ cell-tumor immunosurveillance $(32,33)$. Therefore, to gain more insight into the mechanism of CD226-dependent control of MM by NK cells and $\mathrm{CD} 8^{+} \mathrm{T}$ cells, we analyzed MM burden in 
A
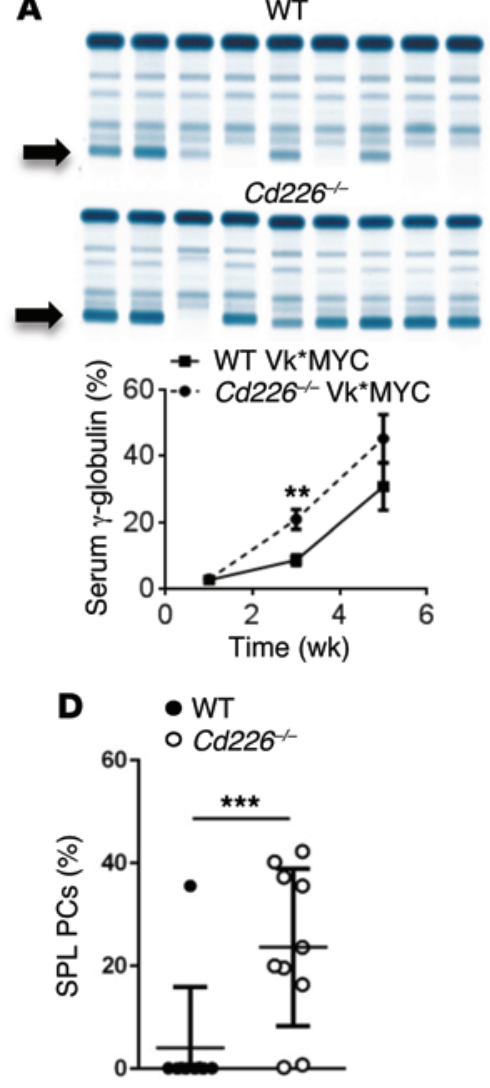

G
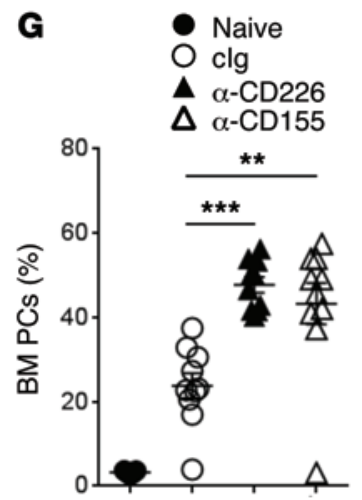

B

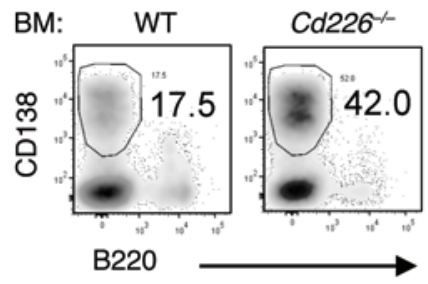

Spleen:

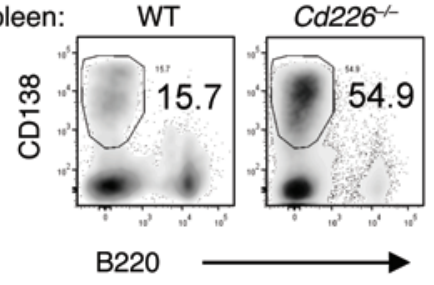

E

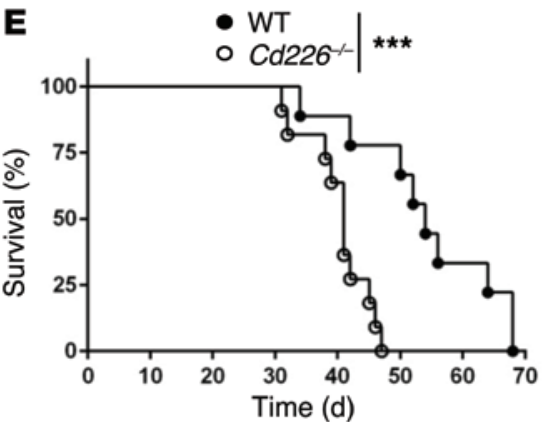

C
$\Delta \Delta \mathrm{Vk}^{*} \mathrm{MYC}$

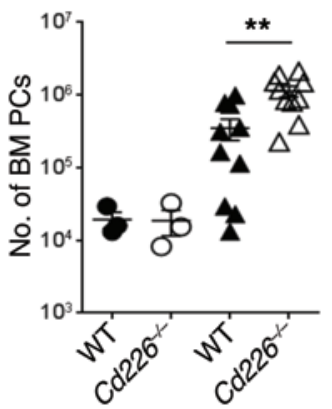

$\bullet \quad$ Naive $\bullet \quad$ Naive

$\Delta \Delta \mathrm{Vk}^{*} \mathrm{MYC}$

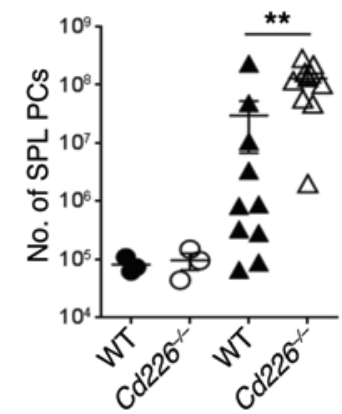

$\mathbf{F}$
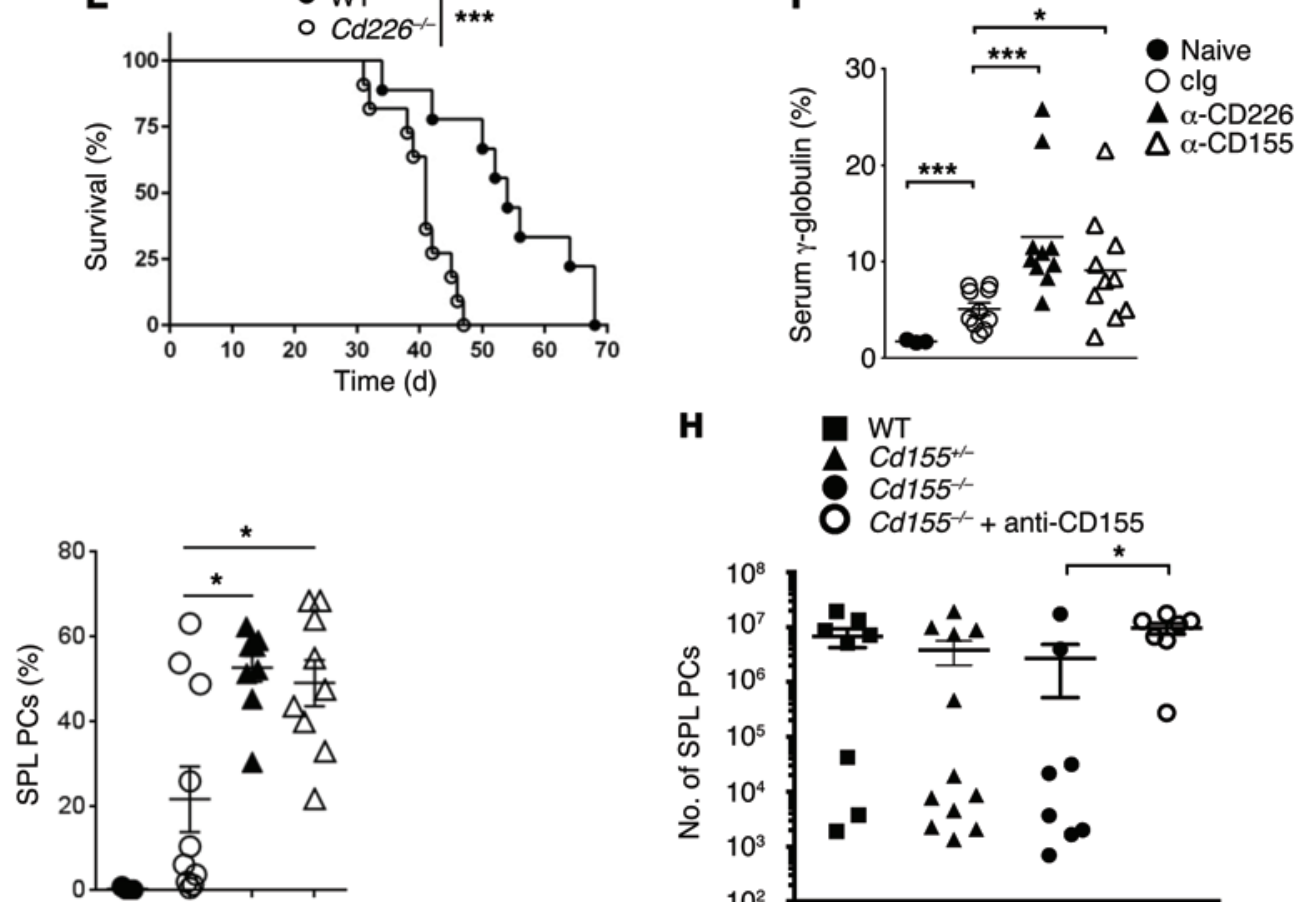

H
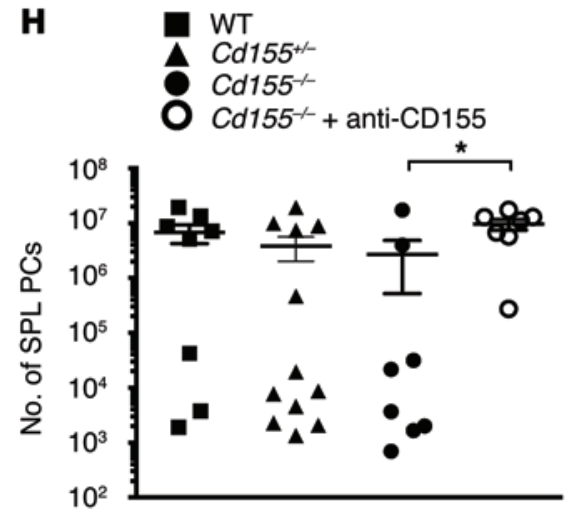

Figure 2. The interaction between CD226 and tumor CD155 is required for the immune-control of MM. (A-C) WT or Cd226 $6^{-/-}$mice were challenged i.v. with $2 \times 10^{6} \mathrm{Vk} 12653$ cells. (A) Representative SPEP and graph showing the mean $\gamma$-globulin level \pm SEM in the serum of the indicated strain of mice. Arrows indicate M-spike. (B) Representative FACS plots showing the frequency of CD138+B220- PCs in the BM and the spleen 5 weeks after injection. (C) Graphs showing the mean number of PCs in the BM and the spleen. Representative experiment out of 4 involving groups of $n=10$ mice. (D and E) WT or Cd222 ${ }^{-1-}$ mice were challenged i.v. with $4 \times 10^{5} \mathrm{Vk12598}$ MM cells. Graphs showing the percentage of malignant PCs was in the spleen (SPL) 3 weeks after injection (D) and the survival of mice over time (E). Representative experiment out of 2 involving groups of $n=10$ mice. (F and G) WT mice were injected with anti-CD226, anti-CD155, or control lgG (clg) before Vk12653 MM cell injection. (F) Mean serum $\gamma$-globulin level \pm SEM 3 weeks after MM injection. (C) Graphs showing the percentages

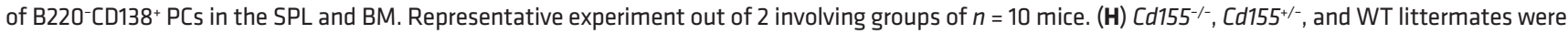
injected with anti-CD155 or control lgG before Vk*MYC challenge. Graph showing the number of B220-CD138+ PCs in the SPL. Data are from 2 pooled experiments involving groups of $n=7-14$ mice. Each symbol represents 1 individual mouse. ${ }^{*} P<0.05,{ }^{* *} P<0.01,{ }^{* * *} P<0.001$. Kruskall-Wallis test was used for multiple comparisons, Mann-Whitney $U$ test was used for single comparison between groups, and Mantel-Cox test was used for survival analysis.

mice deficient of perforin $\left(\mathrm{Pfp}^{-/}\right)$and IFN- $\gamma\left(\mathrm{Ifng} / \mathrm{f}^{-}\right)$. Within 1 week after Vk12653 MM cell injection, we detected higher paraprotein levels in $\mathrm{Pfp}^{-/-}$mice than in Ifng $\mathrm{g}^{-/}$and WT mice (Figure 3B). After 3 weeks, both $P f p^{-/}$and $I f n g^{-/}$mice had higher paraprotein levels than WT mice (Figure 3B). Finally, while no significant differences were observed after 5 weeks between WT and $\mathrm{Pfp}^{-/-}$mice, the
M-spike levels were significantly higher in Ifng $/$ mice (Figure 3B), suggesting that perforin and IFN- $\gamma$ both participate in the antimyeloma immune response, but at slightly different times. Perforin and IFN- $\gamma$ may also have slightly different roles depending on the location of the MM. Indeed, we found that the antimyeloma effects of perforin were restricted to the $\mathrm{BM}$, where $P f p^{-/}$mice contained 
A$$
\text { A }
$$$$
\text { - clg }
$$$$
\text { Anti-AsGM1 }
$$$$
\text { A Anti-CD8 }
$$$$
\triangle \text { Anti-AsGM1 + anti-CD8 }
$$

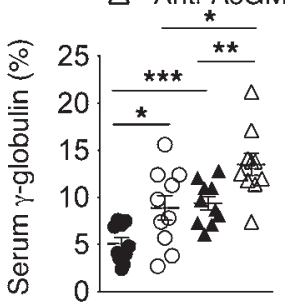
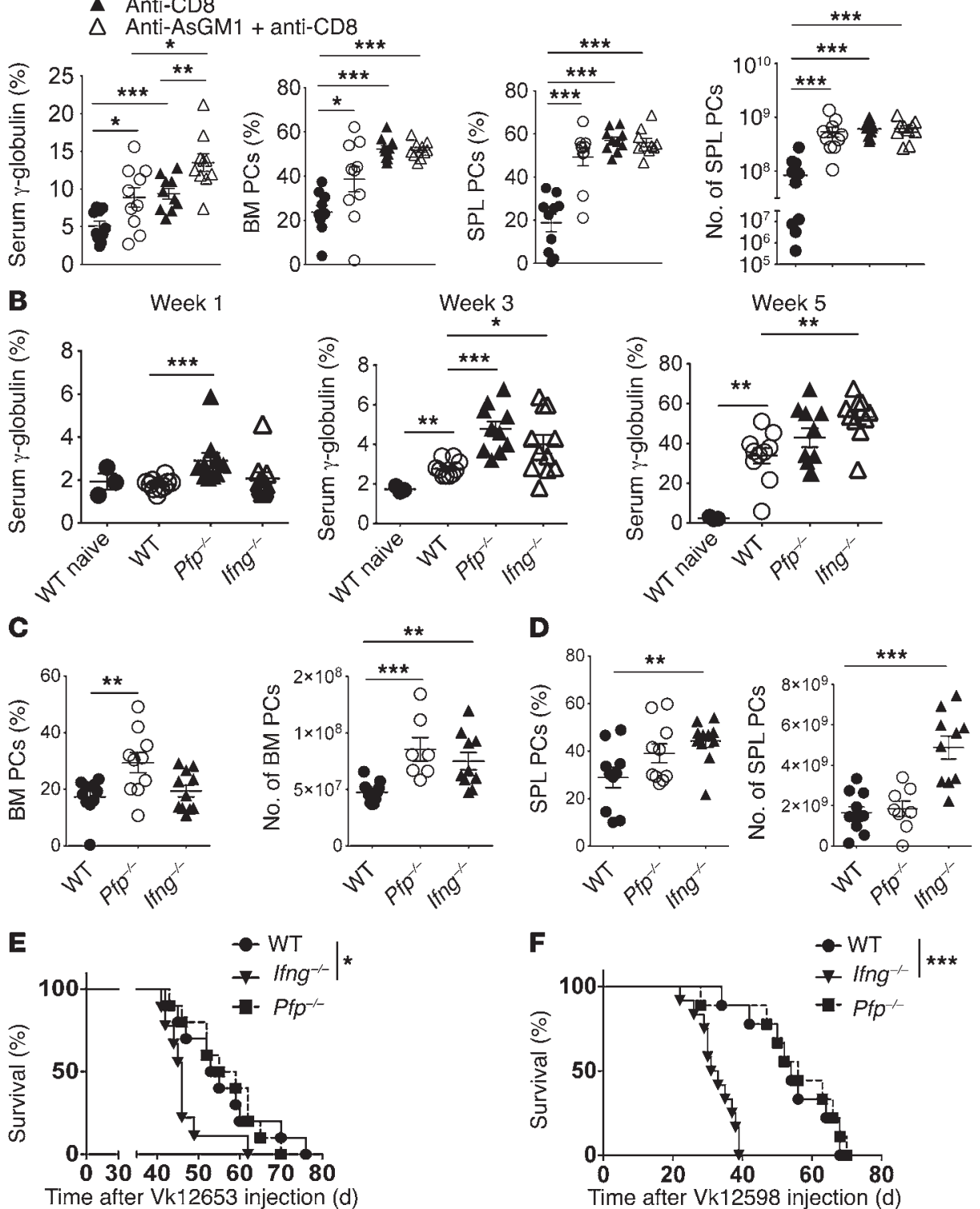

Figure 3. Critical role for NK and CD8 ${ }^{+} \mathrm{T}$ cells in the control MM through perforin and interferon- $\gamma$ pathways. (A) NK cells and $C D 8^{+} T$ cells limit $V k^{*}$ MYC MM burden in vivo. WT mice were injected with control IgG, anti-AsGM1 and/or anti-CD8 $\beta$ to deplete NK cells and/or CD8 ${ }^{+} \mathrm{T}$ cells, and were challenged with Vk12653 cells. The $\gamma$-globulin levels in the serum of the indicated groups of mice 3 weeks after injection, and the percentages and numbers of $\mathrm{CD} 138^{+} \mathrm{B} 220^{-} \mathrm{PCs}$ in the BM and in the spleen (SPL) of the indicated groups of mice are shown. Representative experiment out of 2 involving groups of $n=10$ mice. (B-D) $\mathrm{WT}, \mathrm{Pfp}^{-1-}$, and $\mathrm{Ifng} \mathrm{fl-}^{-1}$ mice were injected i.v. with $2 \times 10^{6} \mathrm{Vk} 12653$ cells. (B) MM burden was evaluated by SPEP 1,3 , and 5 weeks after injection. The $\gamma$-globulin levels in the serum of the indicated groups of mice are shown. ( $\mathbf{C}$ and $\mathbf{D}$ ) the percentages and numbers of CD138+B220- PCs in the BM (C) and in the SPL (D) of the indicated groups of mice are shown. Representative experiment out of 2 involving groups of $n=10$ mice. (E and F) WT, $\mathrm{Pfp}^{-/-}$, and $/ \mathrm{fng}^{-/-}$mice were injected i.v. with $5 \times 10^{5} \mathrm{Vk} 12653$ cells $(\mathbf{E})$ or $4 \times 10^{5} \mathrm{Vk} 12598$ cells $(\mathbf{F})$, and the survival of mice was analyzed after injection. Groups of $n=10$ mice are shown. Each symbol represents 1 individual mouse. ${ }^{*} P<0.05$, ${ }^{* *} P<0.01$, ${ }^{* *} P<0.001$; Mann-Whitney $U$ test (A-D) and Mantel-Cox test (E and $\mathbf{F}$ ). a higher fraction of $\mathrm{B}^{2} 2 \mathrm{O}^{-} \mathrm{CD} 138^{+} \mathrm{PCs}$ than WT mice (Figure $3 \mathrm{C}$ ). By contrast, $\mathrm{Ifng}^{-/}$mice had higher spleen weight and contained significantly higher percentages and numbers of MM cells in the spleen than WT and $P f p^{-/}$mice (Figure 3D and Supplemental Figure 4). Finally, we found that IFN- $\gamma$-deficient mice injected with Vk12653 or Vk12598 MM cells had a significantly shorter survival than WT or $P f p^{-1-}$ mice (Figure 3, E and F). Therefore, although perforin and IFN- $\gamma$ are both involved in the immune control of MM, the impact of IFN- $\gamma$ deficiency was more pronounced, reinforcing the importance of this pathway in disease control by immune cells.

Progressive immune defects are associated with $M M$ progression in vivo. MM has been associated with a progressive dysfunction of NK cells and CD $8^{+} T$ cells $(10,11)$. To better understand the impact of myeloma progression on host immune responses, we first analyzed immune populations during MM development in vivo. $\mathrm{Vk}^{*} \mathrm{MYC}$ mice bearing spontaneous MM and nontransgenic control mice were bled at 600 and 700 days, and peripheral blood was analyzed by flow cytometry. We observed a significant decrease in $\mathrm{CD} 8^{+} \mathrm{T}$ cell and $\mathrm{NK}$ cell percentages in the blood of $\mathrm{Vk}^{*} \mathrm{MYC}$ mice as compared to nontransgenic mice (Figure 4, A and B). A similar decrease in NK cells and $\mathrm{CD} 8^{+} \mathrm{T}$ cells was observed in the $\mathrm{BM}$ of mice injected with Vk12653 MM cells, as compared to noninjected mice (Figure 4C). Finally, we found an inverse correlation between the number of BM PCs and the percentage of NK cells and CD8 $8^{+} \mathrm{T}$ cells, demonstrating that MM burden is associated with a decrease in the percentage of NK and $\mathrm{CD} 8^{+} \mathrm{T}$ cells in the BM (Figure 4D). We next analyzed CD226 expression on CD8 ${ }^{+} \mathrm{T}$ cells and NK cells and observed that, in both spontaneous and transplanted $\mathrm{Vk}^{*} \mathrm{MYC}$ models, a decrease in cell surface expression of CD226 on CD8 ${ }^{+} \mathrm{T}$ cells and NK cells was associated with MM progression (Figure 4, $\mathrm{E}$ and $\mathrm{F}$ ). In addition, the number of BM PCs was inversely correlated to the expression of $\mathrm{CD} 226$ on $\mathrm{NK}$ and $\mathrm{CD} 8^{+} \mathrm{T}$ cells (Figure $4 \mathrm{G})$. Altogether, our results show that progressive immune defects are associated with MM development. 
$\mathbf{A}$

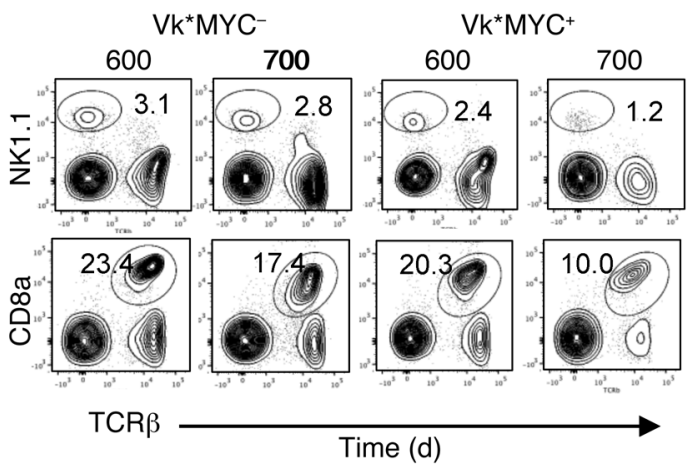

B

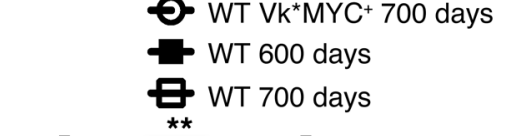

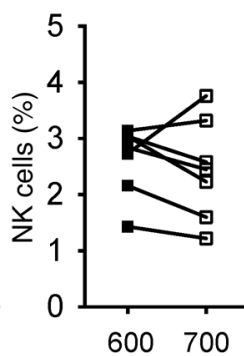
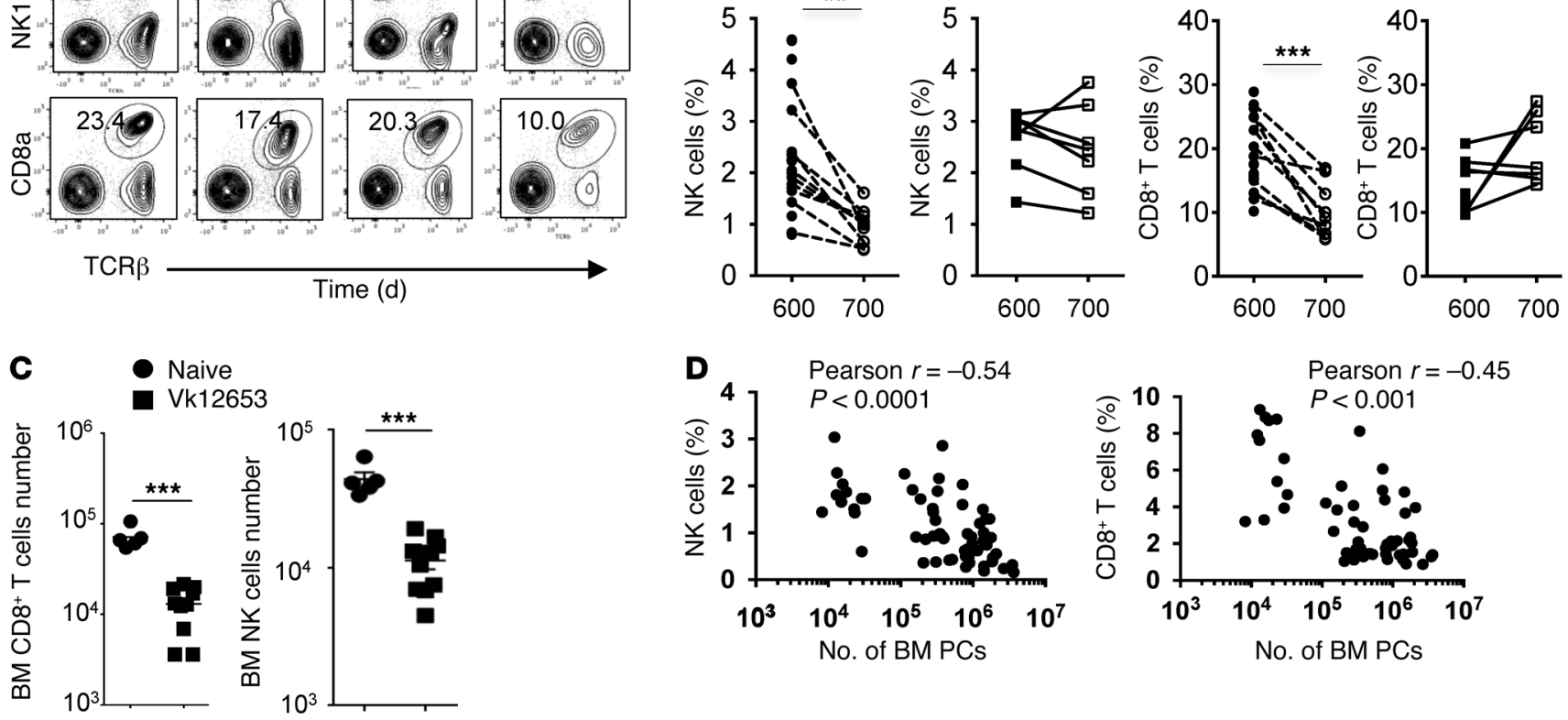
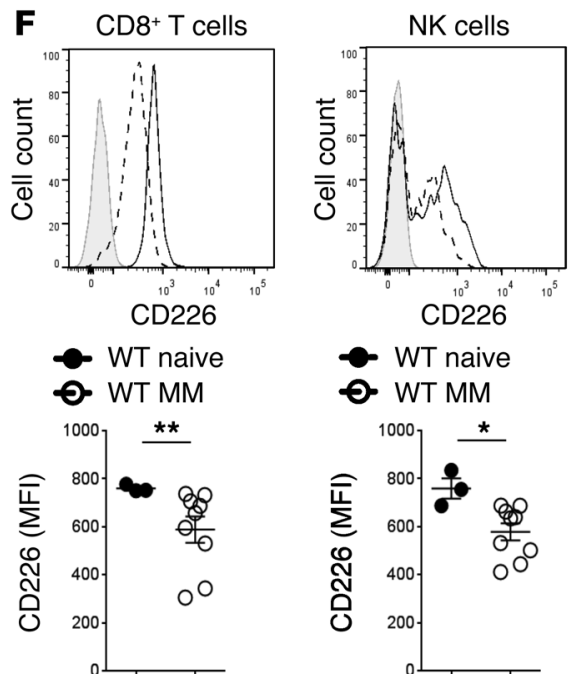
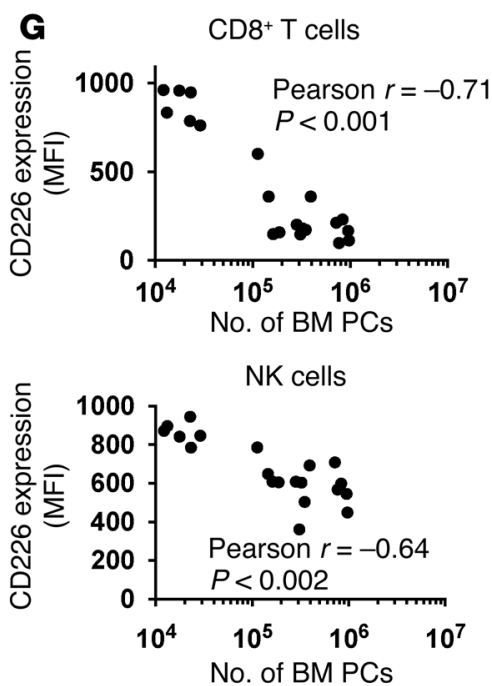

Figure 4. Progressive immunosuppression is associated with myeloma progression in vivo. (A and B) Representative FACS plots (A) and graphs (B) showing the percentages of TCR $\beta^{+}$CD8 $8^{+}$cells and NK1.1 $1^{+}$TCR $\beta^{-}$NK cells in the peripheral blood of $C d 226^{+/+} \mathrm{Vk} k^{*}$ MYC mice $(n=20)$ and nontransgenic control mice $(n=7)$ at 600 and 700 days. (C) Graphs showing the numbers of TCR $\beta^{+} C D 8^{+}$cells and NK1.1+TCR $\beta^{-}$NK cells in the BM of naive mice or mice injected with Vk12653 MM cells for 5 weeks. Representative experiment out of 2 involving groups of $n=5-10$ mice. (D) Graphs showing the correlation between the percentage of NK and CD8 ${ }^{+} \mathrm{T}$ cells and the number of BM PCs in the BM of mice injected with Vk12653 cells for 5 weeks $(n=61)$. Pearson $r$ coefficient and associated $P$ values are shown. (E) Graphs showing the mean fluorescence intensity (MFI) of CD226 on CD8 ${ }^{+}$T cells and NK cells as in $\mathbf{A}$. (F) Representative histogram plots and graphs showing the MFI of CD226 staining on NK cells and CD8 $8^{+}$cells as in C. Isotype control staining (gray line), and CD226 staining on naive mice (black line) and MM-bearing mice (dotted line) are shown. (G) Graph showing the correlation between the expression of CD226 on NK cells and $\mathrm{CD}^{+} \mathrm{T}$ cells and the number of BM PCs in the BM of mice injected with Vk12653 cells for 5 weeks $(n=20)$. Pearson $r$ coefficient and associated $P$ values are shown. Each symbol represents 1 individual mouse. ${ }^{*} P<0.05,{ }^{* *} P<0.01,{ }^{* *} P<0.001$; paired Student's $t$ test (B and $\left.\mathbf{E}\right)$ and Mann-Whitney $U$ test $(\mathbf{C}$ and $\mathbf{F}$ ).

Optimal antimyeloma therapy requires CD226. It is now well established that the in vivo antitumor efficacy of chemotherapy can rely on the activation of the immune system $(34,35)$. However, in the absence of a mouse myeloma model, the role of immune components in the clinical efficacy of antimyeloma drugs such as Btz and cyclophosphamide (CTX) remained to be determined.
To address this question, cohorts of WT and $C d 226^{-/-}$mice were injected with Vk12653 MM cells. After 3 weeks, when mice started to develop measurable M-spike, mice were injected either with $\mathrm{cIg}$ or anti-CD226 mAbs and were subsequently treated with PBS, Btz (i.p.; $0.5 \mathrm{mg} / \mathrm{kg}$ ), or CTX (i.p.; $50 \mathrm{mg} / \mathrm{kg}$ ) twice a week (Supplemental Figure 5). Evolution of tumor burden was assessed every week by 

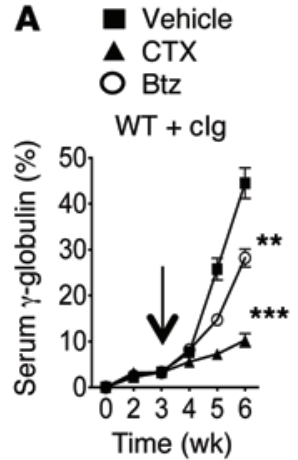

C

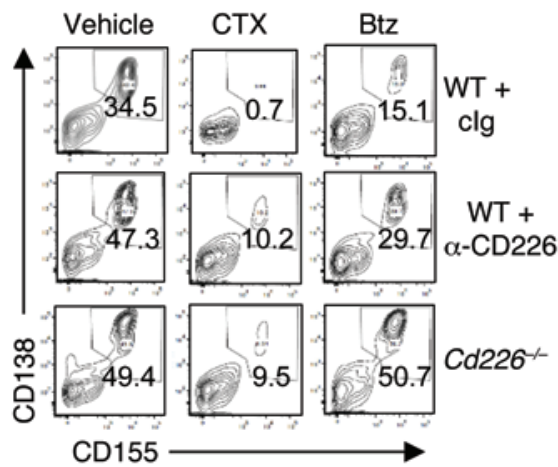

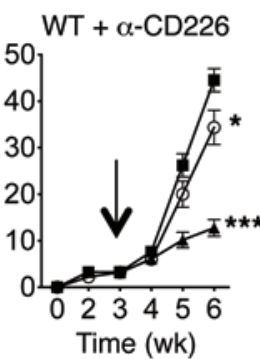

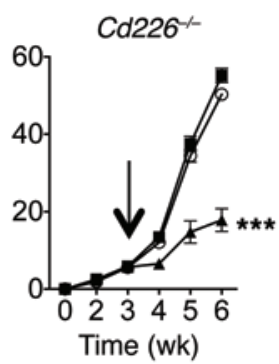

D

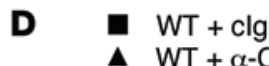

A $\mathrm{WT}+\alpha-\mathrm{CD} 226$

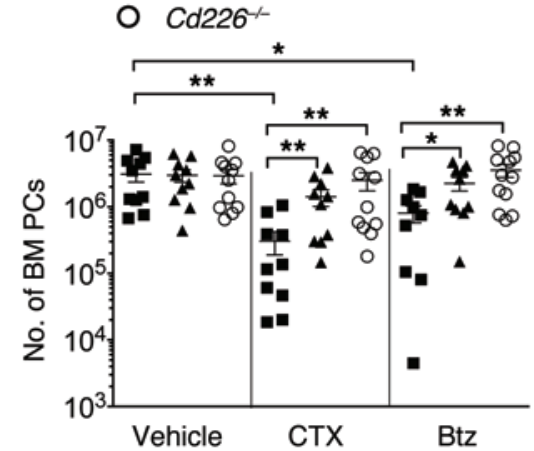

B $\quad \mathrm{WT}+\mathrm{clg}$

A $W T+\alpha-C D 226$

O $\mathrm{Cd}_{222}^{--}$

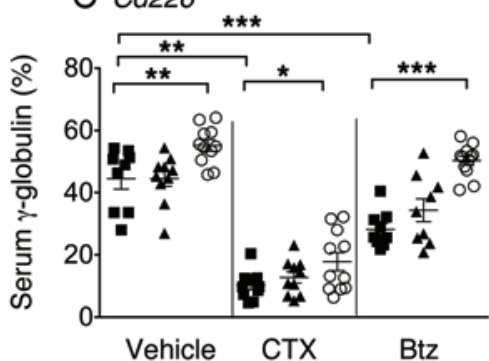

Figure 5. Optimal antimyeloma therapy requires CD226. (A-E) The therapeutic efficacy of Btz and CTX is altered in the absence of CD226. (A-D) WT and $\mathrm{Cd} 226^{-/-}$mice were injected i.v. with $2 \times 10^{6} \mathrm{Vk} 12653 \mathrm{MM}$ cells. After 3 weeks (indicated by arrows), mice were injected either with IgC or anti-CD226 mAbs and were subsequently treated with vehicle (PBS), Btz (i.p.; $0.5 \mathrm{mg} / \mathrm{kg}$ ), or CTX (i.p.; $50 \mathrm{mg} / \mathrm{kg}$ ) twice a week. (A and B) Serum paraproteinemia was analyzed every week by SPEP. Graphs showing the mean $\gamma$-globulin percentages \pm SEM in the indicated groups of mice over time (A) and after 3 weeks of treatment (B). (C and $\mathbf{D})$ Representative FACS plot showing the percentages (C) and graphs showing the numbers (D) of malignant CD138 ${ }^{+}$CD155 $5^{+}$PCs in the $\mathrm{BM}$ of the indicated groups of mice at the end of the treatment. Data are pooled from 2 independent experiments involving 9 groups of $n=9-12$ mice. Each symbol represents 1 individual mouse. (E) WT and $C d 226^{-/-}$mice were injected i.v. with $4 \times 10^{5} \mathrm{Vk} 12598 \mathrm{MM}$ cells. After 1 week, mice were treated with PBS or Btz (i.p.; $0.5 \mathrm{mg} / \mathrm{kg}$ ) twice a week for 4 weeks, and the survival of the indicated groups of mice was monitored. Experiment involving groups of $n=10$ mice. ${ }^{*} P<0.05,{ }^{* *} P<0.01$, ${ }^{* * *} P<0.001$; Mann-Whitney $U$ test (A, B, and $\left.\mathbf{D}\right)$ and Mantel-Cox test (E).

SPEP (Figure 5, A and B). CTX was very effective in limiting tumor progression. All groups of mice (WT mice plus Ig, WT mice plus anti-CD226, or $C d 226^{-/}$mice) had almost undetectable M-spike after 3 weeks of treatment, while vehicle-treated mice all developed severe paraproteinemia (Figure 5, A and B). Vk12653 cells have been selected for increased resistance to Btz treatment $(30,31)$. Indeed, we found that Vk12653 cells were less sensitive to Btz than CTX, but we still observed a significant reduction in $\gamma$-globulin levels in WT mice treated with Btz (Figure 5, A and B). Surprisingly, in Cd226 mice or WT mice injected with anti-CD226 mAbs, Btz treatment failed to limit $\mathrm{M}$-spike progression, suggesting that the absence of CD226 may affect the efficacy of Btz treatment (Figure 5, A and B).

The spleen and BM from different groups of mice were then collected after 3 weeks of treatment and analyzed by FACS for MM cell infiltration. All groups of vehicle-treated mice reached end point disease and had massive tumor infiltration in the BM and the spleen (Figure 5, C and D, and Supplemental Figure 5). WT mice treated with CTX had almost undetectable $\mathrm{CD} 138^{+} \mathrm{CD} 155^{+}$malignant PCs in the BM and the spleen. WT mice injected with anti-CD226 mAbs or $\mathrm{Cd}_{226^{-/}}$mice also had a drastic decrease in the percentage and number of tumor cells in the BM and the spleen after CTX treatment, but in contrast to WT mice, a bigger residual population of $\mathrm{CD} 138^{+} \mathrm{CD} 155^{+}$malignant PCs was still detectable in the absence of
CD226 in both the BM and the spleen (Figure 5, C and D, and Supplemental Figure 5). As a consequence, CTX-treated $C d 226^{-/-}$mice quickly succumbed to MM after the end of the treatment, unlike CTX-treated WT mice that survived significantly longer than vehicle-treated mice (Supplemental Figure 5). Btz therapy also induced a significant decrease in tumor burden in WT mice, although it was less effective in limiting MM progression than CTX (Figure 5, C and D, and Supplemental Figure 5). Surprisingly, the percentages and the numbers of $\mathrm{CD} 138^{+} \mathrm{CD} 155^{+}$tumors in the $\mathrm{BM}$ and spleen of $C d 226^{--}$mice or anti-CD226-injected WT mice were not significantly reduced after Btz treatment, suggesting that the in vivo efficacy of this agent requires an intact CD226 pathway (Figure 5, C and D, and Supplemental Figure 5). To confirm these findings, we performed similar experiments using another $\mathrm{Vk}^{*} \mathrm{MYC}$ cell line. While Btz treatment significantly increased the survival of WT mice injected with Vk12598 MM cells, we observed that its therapeutic effect was compromised in $\mathrm{Cd} 226^{-/}$mice (Figure 5E). Altogether, these results demonstrate that the immune system affects the in vivo efficacy of the antimyeloma drugs CTX and Btz.

Therapeutic efficacy of anti-CD137 immunotherapy against myeloma. Harnessing the immune system to fight tumors has recently proven to be a successful strategy to treat cancer patients (36). mAbs blockinglymphocyte inhibitory receptors programmed death 1 (PD-1; 
A
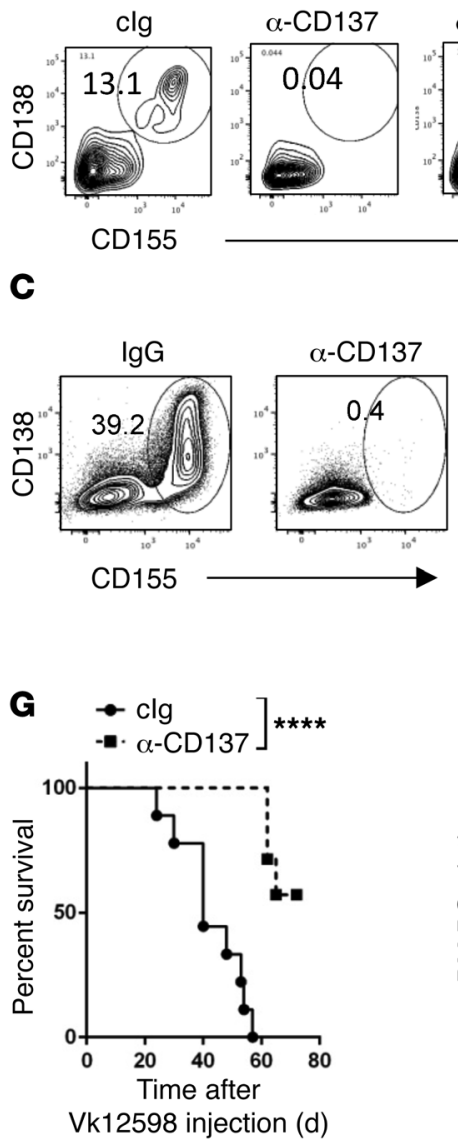

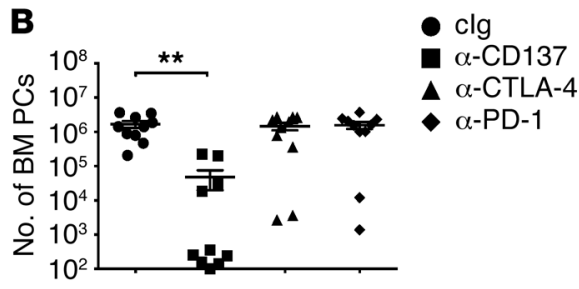

D $\mathrm{clg}$

$\nabla_{\alpha-C D 137}$
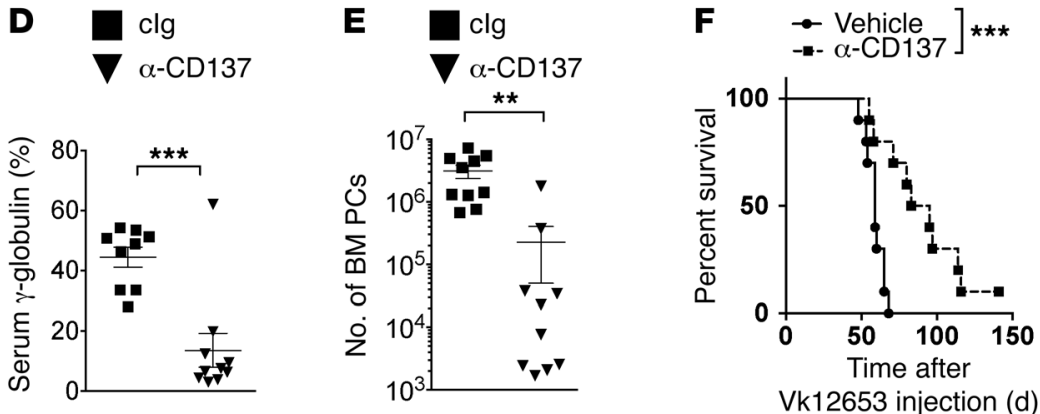

H

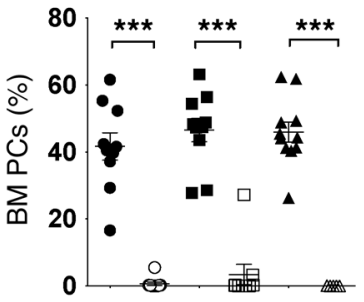

- WT clg

O WT $\alpha$-CD137

- WT $\alpha$-CD226 clg

WT $\alpha$-CD226 $\alpha$-CD137

- Cd226- $\mathrm{clg}$

$\triangle \mathrm{Cd} 226^{-1} \alpha-\mathrm{CD} 137$

Figure 6. Therapeutic efficacy of anti-CD137 immunotherapy against myeloma. (A and B) WT mice were injected with clg, anti-CD137, anti-PD-1, or anti-CTLA-4 and subsequently challenged with $2 \times 10^{6} \mathrm{Vk} 12653 \mathrm{MM}$ cells. Representative FACS plot showing the percentages (A) and graphs showing the numbers (B) of $\mathrm{CD} 138^{+} \mathrm{CD} 155^{+} \mathrm{PCs}$ in the BM at the end of the treatment. Data are representative of 2 independent experiments involving groups of $n=10$ mice. (C-F) WT mice were injected i.v. with $2 \times 10^{6}$ Vk12653 MM cells. After 3 weeks, mice were injected either with IgC or anti-CD137 mAbs. Representative FACS plot and graph showing the percentages (C), the mean $\gamma$-globulin percentages (D), the mean numbers \pm SEM of malignant CD138 ${ }^{+} C D 155^{+} P C s$ in the BM (E) and the survival (F) of the indicated groups of mice. Each symbol represents 1 individual mouse. (G) WT mice were injected i.v. with $4 \times 10^{5} \mathrm{Vk} 12598$ MM cells. After 1 week, mice were treated with IgG or anti-CD137 mAb twice a week for 4 weeks, and their survival was monitored. Experiment involving groups of $n=10$ mice. (H) WT and $C d 226^{-/-}$mice were injected i.v. with $2 \times 10^{6} \mathrm{Vk} 12653 \mathrm{MM}$ cells. After 3 weeks, mice were injected either with IgG or antiCD226 mAb and were subsequently treated with clg or anti-CD137. Graphs show the percentages and the mean numbers \pm SEM of malignant CD138 ${ }^{+}$CD155 ${ }^{+}$ $\mathrm{PC}$ in the BM at the end of the treatment. Data are pooled from 2 independent experiments. ${ }^{* *} P<0.01,{ }^{* * *} P<0.001,{ }^{* * *} P<0.0001 ;$ Mann-Whitney $U$ test (B, D, E, and $\mathbf{H}$ ) and Mantel-Cox test (F and $\mathbf{G})$.

CD279) and CTLA-4 (cytotoxic T lymphocyte antigen 4; CD152) or stimulating coreceptor CD137 (4-1BB, TNFRSF9) are among the most promising therapies currently in the clinic (37-39). However, due to the lack of reliable MM models, the efficacy of such therapies remain unclear in MM. To answer such questions, WT mice were prophylactically injected with cIg, anti-CD137, anti-PD-1, or antiCTLA-4, and subsequently challenged with $\mathrm{Vk}^{*} \mathrm{MYC}$ MM cells. We could not detect any significant differences in tumor burden between control mice treated with anti-PD-1, anti-CTLA-4, and cIg (Figure 6, A and B, and Supplemental Figure 6). By contrast, a striking reduction in the number and percentages of malignant PCs were observed in mice treated with anti-CD137 mAbs (Figure 6, A and B, and Supplemental Figure 6). We next analyzed the antimyeloma effect of anti-CD137 mAbs as a therapeutic agent. Cohorts of WT mice were injected with Vk12653 MM cells. After 3 weeks, when mice started to develop measurable M-spike, mice were injected either with cIg or anti-CD137 mAbs. Analysis of serum Ig levels and PC content in the BM and the spleen after 3 weeks of treatment revealed that anti-CD137 mAbs induced a drastic reduction in myeloma burden in most of the mice treated (Figure 6, C-E). Long-term survival analyses revealed that anti-CD137 mAb-treated mice have a significantly longer survival than PBS-treated mice (Figure 6F). These findings were confirmed in another in vivo model of MM, since we observed that anti-CD137 treatment also significantly increased the survival of WT mice injected with Vk12598 MM cells (Figure 6G). Surprisingly, anti-CD137 treatment also led to a significant reduction of MM development in $\mathrm{Cd} 226^{--}$mice or in mice treated with anti-CD226 mAbs (Figure 6H and Supplemental Figure 6), suggesting that the presence of functional CD226 is dispensable for the antimyeloma response to anti-CD137 mAb immunotherapy.

Anti-CD137 immunotherapy against myeloma requires NK and $C D 8^{+} T$ cells. To gain more insight into the mechanism involved in the antimyeloma effect of anti-CD137 mAb, we performed a phenotypic analysis of immune cells infiltrating the BM of Ig-treated 
A
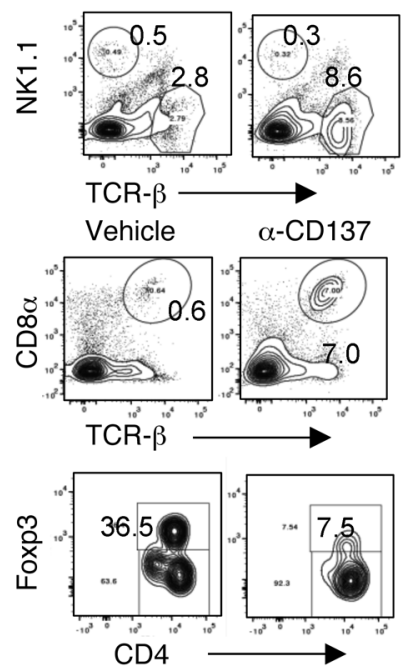

B $\mathrm{clg}$
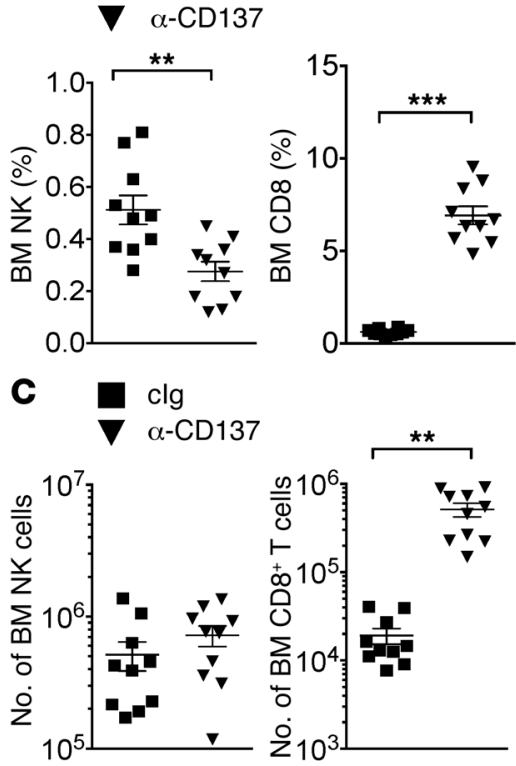
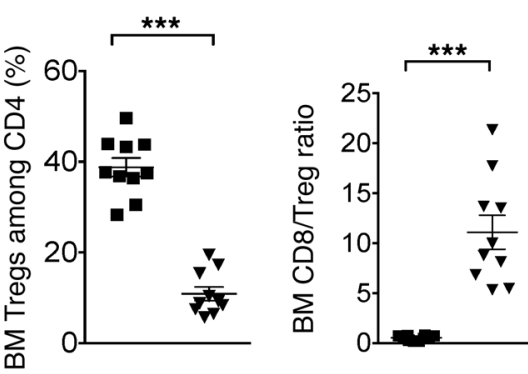
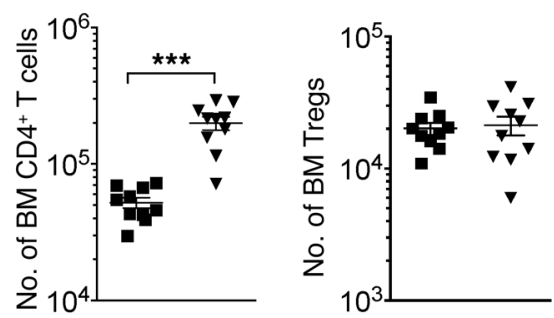

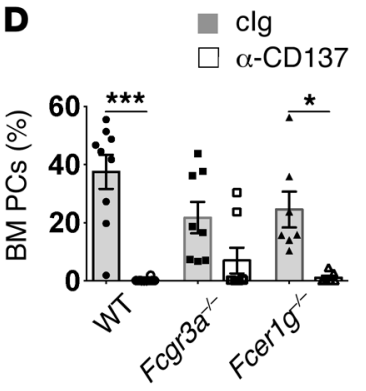

$\mathbf{F}$
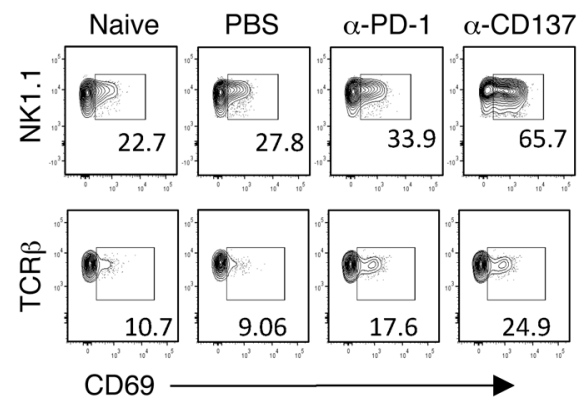

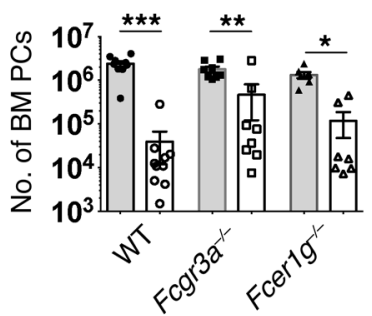

G

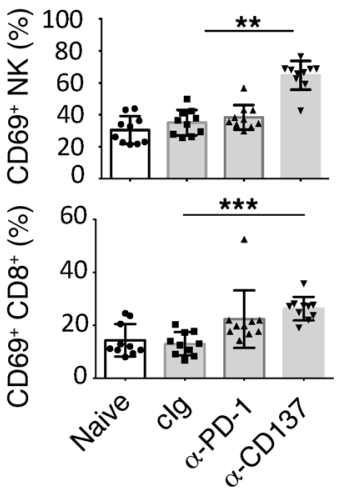

E $\quad \mathrm{clg}$

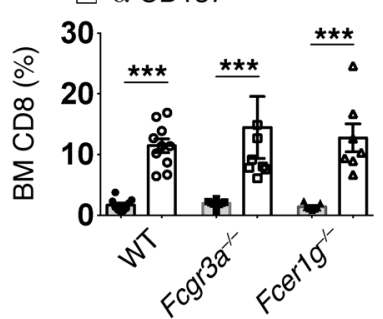

H

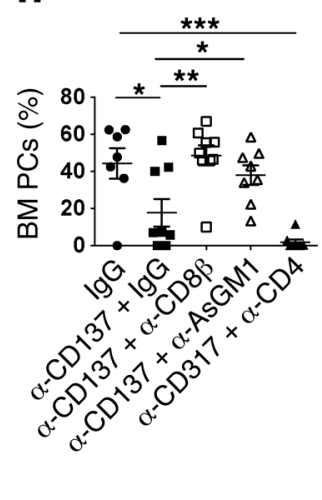

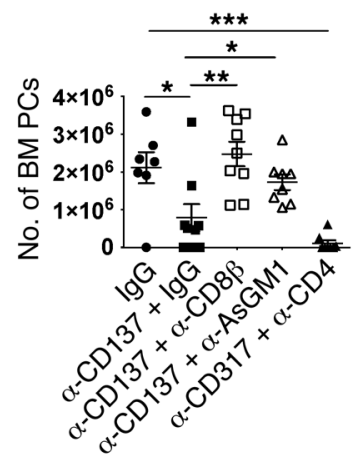

Figure 7. Mechanism of anti-CD137 immunotherapy against myeloma. (A-H) The indicated groups of mice were injected i.v. with $2 \times 10^{6} \mathrm{Vk} 12653 \mathrm{MM}$ cells. After 2-3 weeks, mice with established MM were injected either with IgG, anti-CD137, or anti-PD-1 mAbs. (A-C) Representative FACS plot (A) and graphs showing the percentages (B) and the number (C) of the indicated populations of immune cells after 3 weeks of treatment with clg or anti-CD137. Data are pooled from 2 independent experiments. ( $D$ and $\mathbf{E}$ ) Graphs showing the percentages and the number of malignant PCs (D) or CD8 ${ }^{+} T$ cells $(\mathbf{E})$ in the BM of WT, $\mathrm{Fcgr3a}^{-/-}$, or Fcer1g-/- mice treated with IgC or anti-CD137 mAb. (F and $\left.\mathbf{G}\right)$ Representative FACS plot (F) and graphs showing the percentages (G) of NK cells or CD8 ${ }^{+}$T cells expressing CD69 activation marker 6 days after the beginning of the indicated treatment. Data are pooled from 2 independent experiments. (H) Mice were injected with control IgG, anti-CD8 $\beta$, anti-AsGM1, or anti-CD4 to deplete CD8 ${ }^{+}$, NK, or CD4+ cells before anti-CD137 therapy. The percentages and the number of $\mathrm{CD} 138^{+} \mathrm{B} 220^{-} \mathrm{PCs}$ were analyzed by flow cytometry in the BM of the indicated groups of mice at the end of the treatment. Representative experiment out of 2 involving groups of $n=10$ mice. ${ }^{*} P<0.05,{ }^{* *} P<0.01,{ }^{* *} P<0.001$; Mann-Whitney $U$ test.

or anti-CD137 mAb-treated mice. We observed a robust increase in the number and the percentages of $\mathrm{CD}^{+} \mathrm{T}$ cells infiltrating the $\mathrm{BM}$ after 3 weeks of anti-CD137 mAb treatment, as compared to the cIg-treated mice (Figure 7, A-C, and Supplemental Figure 7). By con-

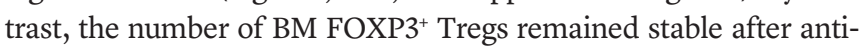

CD137 therapy, while their percentage among BM CD4 ${ }^{+} \mathrm{T}$ cells was significantly decreased (Figure 7, A-C, and Supplemental Figure 7). As a consequence, anti-CD137 therapy induced a strong increase in the ratio between $\mathrm{CD}^{+} \mathrm{T}$ cells and Tregs. Recent evidence suggests that mAb-based immunotherapy can act through the Fc- 
dependent depletion of tumor-associated Tregs (40). We found that a significant fraction of Tregs express CD137 (Supplemental Figure 7), suggesting that a depletion of Tregs by anti-CD137 mAb may be involved in its therapeutic effects. However, Fc receptors were not involved in the antimyeloma effects of anti-CD137 mAbs (of the IgG2a isotype) since $\mathrm{CD}^{+} \mathrm{T}$ cell expansion, as well as BM MM cell burden, was similar in WT mice and mice lacking the $\mathrm{Fc}$ receptor common $\gamma$-chain $\left(F_{c e r} 1 g^{-/}\right)$or the low affinity receptor for IgG2a/ IgG1/IgG2b (Fcgr3a/-) (Figure 7, D and E). These results suggested that anti-CD137 mAbs may directly act on effector cells. Therefore, we analyzed $\mathrm{NK}$ and $\mathrm{CD} 8^{+} \mathrm{T}$ cell activation early after the beginning of the treatment and found that the CD69 activation marker was increased on BM NK cells and CD8 ${ }^{+}$T cells 6 days after CD137 mAb injection (Figure 7, F and G). By contrast, neither NK cells nor $\mathrm{CD}^{+}$ $\mathrm{T}$ cells showed signs of activation after anti-PD-1 therapy, and the $\mathrm{CD}^{+}$cell/Treg ratio remained unchanged, which we have hypothesized to explain the poor therapeutic responses obtained with this treatment in our model (Figure 7, F and G, and Supplemental Figure 7D). We then treated MM-bearing mice with depleting mAbs against NK cells (anti-AsGM1), CD8 ${ }^{+} \mathrm{T}$ cells (anti-CD8 $\beta$ ), or $\mathrm{CD}^{+} \mathrm{T}$ cells (anti-CD4) before anti-CD137 mAb treatment. We found that the anti-CD137 therapy was abrogated in the absence of CD8 ${ }^{+} \mathrm{T}$ cells or NK cells (Figure 7H). Conversely, we found that the depletion of $\mathrm{CD}^{+} \mathrm{T}$ cells did not alter the therapeutic effect of anti-CD137 mAbs. Altogether, these results point toward a critical role of NK cells and $\mathrm{CD}^{+} \mathrm{T}$ cells in anti-CD137-mediated antimyeloma activity.

\section{Discussion}

Until now, the lack of reliable mouse models has limited the understanding of immune interactions with MM in vivo. In this studyusing the most clinically relevant mouse model of $\mathrm{MM}, \mathrm{Vk}^{*} \mathrm{MYC}$ transgenic mice - we demonstrated that the immune system limits spontaneous MM burden in vivo. We provided strong evidence that immune control of $\mathrm{MM}$ is mediated by NK cells and $\mathrm{CD} 8^{+} \mathrm{T}$ cells through CD226 interaction, with CD155 expressed on the cell surface of transformed PCs. Moreover, our results demonstrated that standard drugs used in the management of MM, CTX, and Btz also required CD226 for optimal antimyeloma efficacy in vivo. This seminal work suggests that CD226 may play an important role in MM pathogenesis. The translation of such findings in human MM patients and a better comprehension of immune receptor ligands that control the efficacy of antimyeloma therapy may have strong clinical applications.

Although a number of oncogenic events are associated with myeloma evolution, the driving forces that promote the evolution from MGUS to smoldering and active myeloma have remained obscure $(2,41)$. Primary oncogenic events such as IgH translocations and hyperdiploidy have been associated with cell cycle deregulation, PC survival, and MGUS, while secondary $\operatorname{IgH}$ translocations, RAS mutations, and overexpression of MYC and MYC target genes have been associated with progression to active myeloma $(2,42)$. Our study suggests that host immune reaction may represent another key factor involved in the progression of MM. Management of MGUS and smoldering MM mainly involves a "watch and wait" strategy. Our results suggest that immune monitoring and immunotherapy may greatly impact the outcome for patients with a high risk of developing MM.
Oncogene activation is a powerful inducer of cellular stress that not only activates apoptosis or senescence cellular programs (43), but also stimulates immune recognition through the upregulation of NKG2D and CD226 ligands $(25,44-46)$. It has recently been demonstrated in the E $\mu \mathrm{MYC}$ transgenic mouse model that replication stress induced by MYC overexpression is a strong inducer of CD155 expression (47). Our study demonstrates that malignant PCs developing in $\mathrm{Vk}^{*} \mathrm{MYC}$ mice all displayed a high expression of CD155, as compared to normal PCs. Although no formal proof has been established, this suggests that genomic stress associated with MYC overexpression may be at the origin of CD155 expression on transformed PCs in $\mathrm{Vk}^{*} \mathrm{MYC}$ mice. Similar mechanisms may occur in MM patients, since MYC and MYC target genes - as well as CD155 - are often overexpressed in malignant PCs isolated from patients $(8,28)$. Our study suggests that the expression of CD155 may represent a strong determinant of MM immunogenicity, and an extensive understanding of the clinical impact of this ligand expression in MM patients is now required. CD226 was also shown to interact with CD112 $(48,49)$, but the relevance of CD112/CD226 interactions in mice remains unclear (50). Although, CD112 expression was usually low or absent on $\mathrm{Vk}^{*} \mathrm{MYC}$ MM cells, this ligand was found to be expressed on human MM cells and could also play a role in the pathogenesis of this disease (8).

Recent studies indicate that the efficacy of several chemotherapies in vivo relies on the immune system (34). Although most antimyeloma agents are thought to act primarily through direct cytotoxic effects, additional effects on the immune system may contribute to their efficacy in vivo. We observed that the absence of CD226 or neutralization of this receptor affected the antimyeloma activity of CTX and Btz, providing strong evidence that immune components and CD226 may represent strong determinants of antimyeloma treatment efficacy. Low-dose CTX has been shown to deplete Tregs and restore T and NK cell functions (51-54). The depletion or neutralization of Tregs and the reactivation of NK and $\mathrm{CD}^{+}$effector lymphocytes upon CTX treatment may account for the immune-dependent action of CTX in our experiment.

Despite being one of the most active drugs used in the management of MM, malignant PCs often acquire resistance to Btz, leading to patient relapse (29). Therefore, to mimic more closely what may happen in human patients, we chose to identify the role of immune components using Btz-resistant tumor cells, Vk12653 and Vk12598 (30, 31). Although Vk12653 and Vk12598 cells were less sensitive to Btz than CTX, the progression of MM was still significantly affected by Btz treatment in immune-competent mice. However, the antimyeloma efficacy of Btz was completely lost in the absence of CD226, suggesting that the in vivo efficacy of this drug mainly relies on immunity. Such results suggest that immunotherapeutic approaches may greatly enhance the efficacy of this drug in $\mathrm{MM}$, and future experiments will have to determine the underlying mechanisms of the CD226-dependent effects of Btz. Part of the effect of this drug might be mediated through NK cell activation, since Btz was shown to augment the efficacy of adoptively transferred NK cells in both murine tumor models and cancer patients $(55,56)$. Indeed, proteasome inhibitors alter MHC class I (MHC-I) peptide processing machinery, and MM cells treated with Btz have reduced cell surface expression of MHC-I and are more susceptible to NK cell-mediated killing (57). Btz has also been shown to stimu- 
late the expression of CD155 and NKG2D ligands at the cell surface of MM cells (26). CD155 expression is already high on $\mathrm{Vk}^{*} \mathrm{MYC}$ MM cells, but we cannot rule out that an increase in CD155 expression on accessory cells may contribute to the CD226-dependent MM reduction in Btz-treated mice. Alternatively, Btz treatment may also trigger immunogenic cell death of MM cells (34) and stimulate the presentation of tumor antigens to cytotoxic T cells. For example, previous study demonstrated that Btz increases the uptake of dying human myeloma cells by dendritic cells (58).

The inclusion of drugs such as IMiDs and Btz has resulted in a significant prolongation of survival of MM patients. However, MM is still an incurable disease with a median survival not exceeding 5 years in adults (1). Immune stimulatory mAbs have recently emerged as a promising strategy and are currently tested in several malignancies, with the hope of further improving patients' survival (59). Recent clinical trials have demonstrated a significant therapeutic impact of PD- 1 inhibition on a variety of solid tumors $(37,60)$. The broad expression of PD-1/PD-ligand 1 (PD-L1) in the microenvironment of myeloma suggest this pathway may play an important role in the immune evasion by MM cells (61). Several studies demonstrated that PD-1 or PD-L1 blockade increases immune cell reactivity against malignant PCs (62-64). In our model, we could not observe any therapeutic or notable immune stimulatory effect of anti-PD-1 treatment. However, we do not exclude that such a treatment administered with a different schedule or in combination with other immune stimulatory or chemotherapeutic agents may have potent antitumor activity. Indeed, we found that PD-1 expression is very low on spleen or BM effector cells in the early phase of MM development, possibly explaining the low therapeutic activity of this drug in our model. However, we found that PD-1 was highly upregulated on CD8 ${ }^{+} \mathrm{T}$ cells and NK cells following anti-CD137 therapy, suggesting that anti-PD-1 mAbs may combine with anti-CD137 therapy.

Anti-CD137 is one of the most interesting immunostimulatory mAbs tested for cancer therapy (65-69). Currently, BMS-663513, a humanized anti-CD137 antibody, is in clinical trials in patients with solid tumors, including melanoma, renal carcinoma, ovarian cancer, and B cell malignancies (70). Our present results demonstrate that anti-CD137 exerts strong antimyeloma effects, providing a strong rationale for testing this agent in MM. It would now be interesting to analyze the benefit of combining such treatments with classical MM chemotherapy, such as Btz. Although CD226 had a strong impact in the control of MM burden in our model, the antimyeloma effects provided by anti-CD137 mAb therapy appeared independent of CD226. CD137-mediated signals were shown to increase DC maturation, to reverse CD8 T cell anergy, and to render effector $\mathrm{T}$ cells refractory to Treg-mediated suppression (66). Anti-CD137 mAb-mediated T cell activation may therefore bypass the requirement for CD226 costimulatory signals. Indeed, besides its well-known role in cytotoxicity, CD226 has also been shown to play an important role in T cell activation mediated by antigen-presenting cells $(16,22)$, and more recently, decreased CD226/CD155 interactions were shown to be an important feature of exhausted $\mathrm{CD} 8^{+} \mathrm{T}$ cells within human tumors (71). With the progression of MM, CD226 is often downregulated on effector cells (8), and therefore finding an immunotherapeutic agent that allows the targeting of malignant PCs independently of the CD226 pathway is a valuable discovery. Our study suggests that antiCD137 therapy may bypass the potential immune exhaustion associ- ated with MM disease development. Analysis of BM and spleen lymphocyte populations after immunotherapy revealed that anti-CD137 decreased Treg percentages and simultaneously increased $\mathrm{CD} 8^{+} \mathrm{T}$ cells, leading to a dramatic increase in the CD8 $\mathrm{T}$ cell/Treg ratio. Although such findings are reminiscent of the effect of anti-CTLA-4 mAbs in solid tumors (40), unlike anti-CTLA-4, anti-CD137 effects were not mediated through an Fc-dependent depletion of Tregs. Consistent with a previous study (72), we found that anti-CD137 therapy required the presence of both $\mathrm{CD}^{+} \mathrm{T}$ cells or NK cells, while $\mathrm{CD}^{+} \mathrm{T}$ cells were dispensable. Indeed, NK cells and $\mathrm{CD} 8^{+} \mathrm{T}$ cells were activated early after the beginning of anti-CD137 therapy, and their depletion abrogated the therapeutic effect of anti-CD137. MM growth relies on a complex network of cytokines and cellular interactions (27). Future experiments will have to determine whether therapy-induced changes in the BM microenvironment may alter this network and, by this mechanism, limit MM development. It would now be interesting to analyze the benefit of combining such treatments with classical MM chemotherapy, such as Btz.

\section{Methods}

Mice. All mice used in this study were on a C57BL/6 genetic background. C57BL/6 WT mice were purchased from the Walter and Eliza Hall Institute for Medical Research or ARC Animal Resource Centre. $\mathrm{Vk}^{*}$ MYC (Tg[Tgkv3-5-MYC]\#Plbe) (28), Cd226 $6^{-/-}$(16), and Pfp ${ }^{-/}$, Ifng $^{-/}$, and $\mathrm{Rag}^{-/-} \mathrm{Il} 2 \mathrm{rg}^{-/}$mice have already been described (73). Cd155 $5^{-/-}$ mice were provided by Yoshimi Takai (Kobe University, Kobe, Japan). $\mathrm{Fcerlg}^{-/-}$and $\mathrm{Fcgr}^{-a^{-/-}}$mice were provided by Eva Orlowski (Burnet Institute, Melbourne, Australia). All mice were bred and maintained at either the Peter MacCallum Cancer Centre or QIMR Berghofer Medical Research Institute and were used between the ages of 6-14 weeks.

$V k^{*} M Y C$ aging experiments. $\mathrm{Vk}^{*} \mathrm{MYC}$ transgenic mice were crossed with $C d 226^{-/-}$mice. $C d 226^{+/+}(n=55), C d 226^{-/+}(n=27)$, and $C d 226^{-/-}(n=25) \mathrm{Vk}^{*} \mathrm{MYC}$ transgenic littermate controls, as well as $C d 226^{+/+}(n=12), C d 226^{-/+}(n=15)$, and $C d 226^{-/-}(n=9)$ nontransgenic littermate controls, were monitored for MM development and survival during a period of 3 years. The presence of monoclonal Ig (M-spike) and the level of $\gamma$-globulin in the serum were quantified by SPEP (HYDRASYS, Sebia Hydragel) every 2 months. The content of malignant PCs in the BM was determined in the different groups of mice postmortem (Supplemental Figure 1). A second cohort of $C d 226^{+/+}(n=15), C d 226^{-/}(n=14) \mathrm{Vk}^{*} \mathrm{MYC}$ transgenic mice, $C d 226^{+/+}(n=6)$, and $C d 226^{-/}(n=10)$ nontransgenic littermate controls was culled at 450 days of age to analyze by flow cytometry the

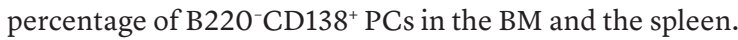

$V k^{*} M Y C$ transplantable model. Transplantable $\mathrm{Vk}^{*} \mathrm{MYC}$ MM cells Vk12653 and Vk12598 were generated as described previously (30). To remove any potential contaminant cells that may derive from the original host, we performed 2 successive transplants by i.v. injection of $2 \times 10^{6} \mathrm{Vk} 12653$ cells or $4 \times 10^{5} \mathrm{Vk} 12598$ cells into $\mathrm{Rag}^{-/-} \mathrm{Il} 2 \mathrm{rg}^{-/}$mice. After the second transplant, all $\mathrm{Rag}^{-{ }^{--}} \mathrm{Il} \mathrm{rg}^{-/-}$mice developed robust M-spikes 5 weeks after injection. Spleen cells from $\mathrm{Rag}^{-1-} \mathrm{Il}_{2 \mathrm{rg}^{-/-}}$mice containing at least $50 \%$ of malignant PCs were frozen and subsequently used as a reliable source of transplantable $\mathrm{Vk}^{*} \mathrm{MYC}$ cells for all our experiments (Supplemental Figure 2). The presence of monoclonal Ig in the serum was determined every 1,3 , and 5 weeks after MM cells injection by SPEP. The percentage and the number of B220$\mathrm{CD} 138^{+} \mathrm{PCs}$ was analyzed in the BM and the spleen by flow cytome- 
try at the end of each experiment. Alternatively, the survival of mice injected with Vk12653 or Vk12598 was monitored over time.

Antimyeloma therapy. For prophylactic treatment, WT or $C d 226^{-1-}$ mice were injected with cIg, anti-CD137 (200 $\mu$ g, 3H3; BioxCell), antiPD-1 (200 $\mu \mathrm{g}$, RMP1-14; BioxCell), or anti-CTLA-4 (200 $\mu \mathrm{g}$, UC104F10; BioxCell) i.p. on days -1 and 0 , and every week after MM cell injection. For a therapy experiment, WT and $C d 226^{-/-}$mice were injected i.v. with $2 \times 10^{6}$ Vk12653 cells. After 3 weeks, when mice started to develop detectable M-spike, mice were randomly assigned into groups that received vehicle (PBS), CTX (i.p. $50 \mathrm{mg} / \mathrm{kg}$, twice a week), Btz (i.p. 0.5 $\mathrm{mg} / \mathrm{kg}$, twice a week), anti-CD137 (100 $\mu$ g, twice a week, 3H3; BioxCell), or anti-PD-1 (100 $\mu$ g, twice a week, RMP1-14; BioxCell). Alternatively, mice injected i.v. with $4 \times 10^{5} \mathrm{Vk} 12598 \mathrm{MM}$ cells were treated with the indicated antimyeloma drugs 1 week after MM cell injection.

Antibody treatments. In vivo antibody depletion and receptor blockade were performed using the following antibodies: control IgG

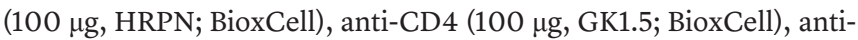

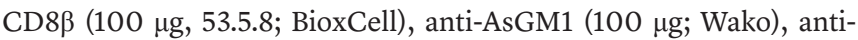
CD226 (200 $\mu \mathrm{g}, 480.1)$, and anti-CD155 (200 $\mu \mathrm{g}, 4.24 .3)$. All antibody injections were given i.p. on days -1 and 0 , and every week after MM injection. Alternatively, antibody injections were given on days -1 and 0 , and every week after the beginning of the antimyeloma therapy.

Flow cytometry. Single-cell suspensions were stained with the following conjugated mAbs: anti-mouse CD3 $\varepsilon$ (145-2C11), TCR $\beta$ (H57597), CD4 (RM4-5), FOXP3 (FJK-16s), CD8 $\alpha$ (53-6.7), NK1.1 (PK136), CD69 (H1.2F3), CD226 (480.1), CD112 (829038), CD155 (4.24.3), B220 (RA3-6B2), CTLA-4 (UC10-4B9), H-2Db (28-14-8), H2-Kb (AF6-88-5), Rae-1 (clone 186107), PD-1(RMP1-30), PD-L1 (10F.9G2), PD-L2 (clone 122), CD137 (17B5), CD137L (TKS-1), and CD138 (clone 281-2). All mAbs were purchased from R\&D Systems, BioLegend, or eBioscience. Flow count beads (BD Biosciences) were added to each sample to determine with accuracy the number of the indicated cell populations. Samples were acquired on a LSR IV Fortessa Flow
Cytometer (BD Biosciences). Analysis was performed with Flowjo software (Tree Star Inc.).

Statistics. Statistical analysis was achieved using GraphPad Prism 6 software. A Kruskall-Wallis test was used for multiple comparisons between groups. When significant differences were found, a 2-tailed nonparametric Mann-Whitney test or unpaired $t$ tests were then used for single comparisons between groups. Comparisons between paired samples were analyzed with 2-tailed paired $t$ tests. A Mantel-Cox test was used for survival analysis. Correlation analyses between variables were performed using Pearson rank correlation test. A $P$ value less than 0.05 was considered significant $\left({ }^{*} P<0.05,{ }^{* *} P<0.01,{ }^{* * *} P<0.001\right)$.

Study approval. All experiments were approved by Peter MacCallum Cancer Centre and QIMR Berghofer Medical Research Institute animal ethics committees.

\section{Acknowledgments}

The authors wish to thank Liam Town, Kate Elder, Joanne Sutton, and Shellie Brown for breeding, genotyping, maintenance, and care of the mice used in this study. We thank Christopher Chan, Ricky Johnstone, and members of his group for providing the original $\mathrm{Vk}^{*} \mathrm{MYC}$ breeding pairs and helpful discussions. We thank Leesa Wockner for helpful advice concerning the statistical analysis of the data. L. Martinet and D.S. Ritchie were supported by a National Health and Medical Research Council (NH\&MRC) of Australia Project grant (1044392). M.J. Smyth and L. Ferrari de Andrade were supported by a NH\&MRC Australia Fellowship (628623) and Program Grant (1013667). L. Martinet was supported by the ARC cancer research foundation (PDF20140601127).

Address correspondence to: Mark J. Smyth, Immunology in Cancer and Infection Laboratory, QIMR Berghofer Medical Research Institute, 300 Herston Road, Herston 4006, Australia. Phone: 61.7.3845.3957; E-mail: mark.smyth@qimrberghofer.edu.au.
1. Palumbo A, Anderson K. Multiple myeloma. N Engl J Med. 2011;364(11):1046-1060.

2. Kuehl WM, Bergsagel PL. Molecular pathogenesis of multiple myeloma and its premalignant precursor. J Clin Invest. 2012;122(10):3456-3463.

3. Avet-Loiseau H, et al. Long-term analysis of the IFM 99 trials for myeloma: cytogenetic abnormalities [t(4;14), del(17p), 1q gains] play a major role in defining long-term survival. J Clin Oncol. 2012;30(16):1949-1952.

4. Schreiber RD, Old LJ, Smyth MJ. Cancer immunoediting: integrating immunity's roles in cancer suppression and promotion. Science. 2011;331(6024):1565-1570.

5. Galon J, et al. Type, density, and location of immune cells within human colorectal tumors predict clinical outcome. Science. 2006;313(5795):1960-1964.

6. Dhodapkar MV, et al. A reversible defect in natural killer $\mathrm{T}$ cell function characterizes the progression of premalignant to malignant multiple myeloma. JExp Med. 2003;197(12):1667-1676.

7. Dhodapkar MV, Krasovsky J, Olson K. T cells from the tumor microenvironment of patients with progressive myeloma can generate strong, tumor-specific cytolytic responses to autologous, tumor-loaded dendritic cells. Proc Natl Acad Sci U S A. 2002;99(20):13009-13013.

8. El-Sherbiny YM, et al. The requirement for DNAM-1, NKG2D, and NKp46 in the natural killer cell-mediated killing of myeloma cells. Cancer Res. 2007;67(18):8444-8449.

9. Carbone E, et al. HLA class I, NKG2D, and natural cytotoxicity receptors regulate multiple myeloma cell recognition by natural killer cells. Blood. 2005;105(1):251-258.

10. Osterborg A, Nilsson B, Bjorkholm M, Holm G, Mellstedt H. Natural killer cell activity in monoclonal gammopathies: relation to disease activity. Eur J Haematol. 1990;45(3):153-157.

11. Jinushi M, et al. MHC class I chain-related protein A antibodies and shedding are associated with the progression of multiple myeloma. Proc Natl Acad Sci U S A. 2008;105(4):1285-1290.

12. Davies FE, et al. Thalidomide and immunomodulatory derivatives augment natural killer cell cytotoxicity in multiple myeloma. Blood. 2001;98(1):210-216.

13. Kronke J, et al. Lenalidomide causes selective degradation of IKZF1 and IKZF3 in multiple myeloma cells. Science. 2014;343(6168):301-305.

14. Quach H, et al. Mechanism of action of immuno- modulatory drugs (IMiDS) in multiple myeloma. Leukemia. 2010;24(1):22-32.

15. Chan CJ, et al. DNAM-1/CD155 interactions promote cytokine and NK cell-mediated suppression of poorly immunogenic melanoma metastases. JImmunol. 2010;184(2):902-911.

16. Gilfillan S, et al. DNAM-1 promotes activation of cytotoxic lymphocytes by nonprofessional antigen-presenting cells and tumors. J Exp Med. 2008;205(13):2965-2973.

17. Lakshmikanth T, et al. NCRs and DNAM-1 mediate NK cell recognition and lysis of human and mouse melanoma cell lines in vitro and in vivo. J Clin Invest. 2009;119(5):1251-1263.

18. Iguchi-Manaka A, et al. Accelerated tumor growth in mice deficient in DNAM-1 receptor. JExp Med. 2008;205(13):2959-2964.

19. Shibuya A, et al. DNAM-1, a novel adhesion molecule involved in the cytolytic function of $\mathrm{T}$ lymphocytes. Immunity. 1996;4(6):573-581.

20. de Andrade LF, Smyth MJ, Martinet L. DNAM-1 control of natural killer cells functions through nectin and nectin-like proteins. Immunol Cell Biol. 2014;92(3):237-244.

21. Chan CJ, et al. The receptors CD96 and CD226 oppose each other in the regulation of natural killer 
cell functions. Nat Immunol. 2014;15(5):431-438.

22. Ramsbottom KM, et al. Cutting edge: DNAX accessory molecule 1-deficient $\mathrm{CD} 8^{+} \mathrm{T}$ cells display immunological synapse defects that impair antitumor immunity. JImmunol. 2014;192(2):553-557.

23. Lozano E, Joller N, Cao Y, Kuchroo VK, Hafler DA. The CD226/CD155 interaction regulates the proinflammatory (Th1/Th17)/anti-inflammatory (Th2) balance in humans. J Immunol. 2013;191(7):3673-3680.

24. Shibuya K, et al. CD226 (DNAM-1) is involved in lymphocyte function-associated antigen 1 costimulatory signal for naive $\mathrm{T}$ cell differentiation and proliferation. J Exp Med. 2003;198(12):1829-1839.

25. Gasser S, Orsulic S, Brown EJ, Raulet DH. The DNA damage pathway regulates innate immune system ligands of the NKG2D receptor. Nature. 2005;436(7054):1186-1190.

26. Soriani A, et al. ATM-ATR-dependent up-regulation of DNAM-1 and NKG2D ligands on multiple myeloma cells by therapeutic agents results in enhanced NK-cell susceptibility and is associated with a senescent phenotype. Blood. 2009;113(15):3503-3511.

27. Anderson KC, Carrasco RD. Pathogenesis of myeloma. Annu Rev Pathol. 2011;6:249-274.

28. Chesi M, et al. AID-dependent activation of a MYC transgene induces multiple myeloma in a conditional mouse model of post-germinal center malignancies. Cancer Cell. 2008;13(2):167-180.

29. Keats JJ, et al. Clonal competition with alternating dominance in multiple myeloma. Blood. 2012;120(5):1067-1076.

30. Chesi M, et al. Drug response in a genetically engineered mouse model of multiple myeloma is predictive of clinical efficacy. Blood. 2012;120(2):376-385.

31. Matthews GM, et al. Preclinical screening of histone deacetylase inhibitors combined with ABT-737, rhTRAIL/MD5-1 or 5-azacytidine using syngeneic $\mathrm{Vk}^{*} \mathrm{MYC}$ multiple myeloma. Cell Death Dis. 2013;4:e798.

32. Smyth MJ, Thia KY, Street SE, MacGregor D, Godfrey DI, Trapani JA. Perforin-mediated cytotoxicity is critical for surveillance of spontaneous lymphoma. JExp Med. 2000;192(5):755-760.

33. Street SE, Cretney E, Smyth MJ. Perforin and interferon-gamma activities independently control tumor initiation, growth, and metastasis. Blood. 2001;97(1):192-197.

34. Obeid M, et al. Calreticulin exposure dictates the immunogenicity of cancer cell death. Nat Med. 2007;13(1):54-61.

35. Kroemer G, Galluzzi L, Kepp O, Zitvogel L. Immunogenic cell death in cancer therapy. Annu Rev Immunol. 2012;31:51-72.

36. Flemming A. Cancer: PD1 makes waves in anticancer immunotherapy. Nat Rev Drug Discov. 2012;11(8):601.

37. Hamid O, et al. Safety and tumor responses with lambrolizumab (anti-PD-1) in melanoma. $N$ Engl JMed. 2013;369(2):134-144.

38. Kaplan MG. Ipilimumab plus dacarbazine in melanoma. N EnglJ Med. 2011;365(13):1256-1257.

39. Ngiow SF, Knight DA, Ribas A, McArthur GA, Smyth MJ. BRAF-targeted therapy and immune responses to melanoma. Oncoimmunology. 2013;2(6):e24462.
40. Simpson TR, et al. Fc-dependent depletion of tumor-infiltrating regulatory $\mathrm{T}$ cells co-defines the efficacy of anti-CTLA-4 therapy against melanoma. J Exp Med. 2013;210(9):1695-1710.

41. Neben K, et al. Progression in smoldering myeloma is independently determined by the chromosomal abnormalities del $(17 \mathrm{p}), \mathrm{t}(4 ; 14)$, gain 1q, hyperdiploidy, and tumor load. J Clin Oncol. 2013;31(34):4325-4332.

42. Affer M, et al. Promiscuous MYC locus rearrangements hijack enhancers but mostly superenhancers to dysregulate MYC expression in multiple myeloma. Leukemia. 2014;28(8):1725-1735.

43. Lowe SW, Cepero E, Evan G. Intrinsic tumour suppression. Nature. 2004;432(7015):307-315.

44. Unni AM, Bondar T, Medzhitov R. Intrinsic sensor of oncogenic transformation induces a signal for innate immunosurveillance. Proc Natl Acad Sci U S A. 2008;105(5):1686-1691.

45. Liu XV, Ho SS, Tan JJ, Kamran N, Gasser S. Ras activation induces expression of Raet1 family NK receptor ligands. J Immunol. 2012;189(4):1826-1834.

46. Chan CJ, Smyth MJ, Martinet L. Molecular mechanisms of natural killer cell activation in response to cellular stress. Cell Death Differ. 2014;21(1):5-14.

47. Croxford JL, et al. ATM-dependent spontaneous regression of early Emu-myc-induced murine B cell leukemia depends on NK and T cells. Blood. 2013;121(13):2512-2521.

48. Bottino C, et al. Identification of PVR (CD155) and Nectin-2 (CD112) as cell surface ligands for the human DNAM-1 (CD226) activating molecule. J Exp Med. 2003;198(4):557-567.

49. Tahara-Hanaoka S, et al. Functional characterization of DNAM-1 (CD226) interaction with its ligands PVR (CD155) and nectin-2 (PRR-2/CD112). Int Immunol. 2004;16(4):533-538.

50. Stanietsky N, et al. Mouse TIGIT inhibits NK-cell cytotoxicity upon interaction with PVR. Eur J Immunol. 2013;43(8):2138-2150.

51. Ghiringhelli F, et al. $\mathrm{CD} 4^{+} \mathrm{CD} 25^{+}$regulatory $\mathrm{T}$ cells suppress tumor immunity but are sensitive to cyclophosphamide which allows immunotherapy of established tumors to be curative. Eur J Immunol. 2004;34(2):336-344.

52. Ghiringhelli F, et al. Metronomic cyclophosphamide regimen selectively depletes $\mathrm{CD} 4{ }^{+} \mathrm{CD} 25^{+}$ regulatory $\mathrm{T}$ cells and restores $\mathrm{T}$ and $\mathrm{NK}$ effector functions in end stage cancer patients. Cancer Immunol Immunother. 2007;56(5):641-648.

53. Lutsiak ME, Semnani RT, De Pascalis R, Kashmiri SV, Schlom J, Sabzevari H. Inhibition of CD4(+)25+ T regulatory cell function implicated in enhanced immune response by low-dose cyclophosphamide. Blood.2005;105(7):2862-2868.

54. Smyth MJ, Teng MW, Swann J, Kyparissoudis $\mathrm{K}$, Godfrey DI, Hayakawa Y. CD $4^{+} \mathrm{CD} 25^{+}$ $T$ regulatory cells suppress NK cell-mediated immunotherapy of cancer. J Immunol. 2006;176(3):1582-1587.

55. Lundqvist A, Berg M, Smith A, Childs RW. Bortezomib treatment to potentiate the antitumor immunity of ex-vivo expanded adoptively infused autologous Natural Killer cells. J Cancer. 2011;2:383-385.

56. Lundqvist A, Yokoyama H, Smith A, Berg M, Childs R. Bortezomib treatment and regula- tory T-cell depletion enhance the antitumor effects of adoptively infused NK cells. Blood. 2009;113(24):6120-6127.

57. Shi J, et al. Bortezomib down-regulates the cellsurface expression of HLA class I and enhances natural killer cell-mediated lysis of myeloma. Blood.2008;111(3):1309-1317.

58. Spisek R, Charalambous A, Mazumder A, Vesole DH, Jagannath S, Dhodapkar MV. Bortezomib enhances dendritic cell (DC)-mediated induction of immunity to human myeloma via exposure of cell surface heat shock protein 90 on dying tumor cells: therapeutic implications. Blood. 2007;109(11):4839-4845.

59. Couzin-Frankel J. Breakthrough of the year 2013. Cancer immunotherapy. Science. 2013;342(6165):1432-1433.

60. Topalian SL, et al. Safety, activity, and immune correlates of anti-PD-1 antibody in cancer. $N$ Engl JMed. 2012;366(26):2443-2454.

61. Atanackovic D, Luetkens T, Kroger N. Coinhibitory molecule PD- 1 as a potential target for the immunotherapy of multiple myeloma. Leukemia. 2014;28(5):993-1000.

62. Hallett WH, Jing W, Drobyski WR, Johnson BD. Immunosuppressive effects of multiple myeloma are overcome by PD-L1 blockade. Biol Blood Marrow Transplant. 2011;17(8):1133-1145.

63. Benson DM, et al. The PD-1/PD-L1 axis modulates the natural killer cell versus multiple myeloma effect: a therapeutic target for CT-011, a novel monoclonal anti-PD-1 antibody. Blood. 2010;116(13):2286-2294.

64. Rosenblatt J, et al. PD-1 blockade by CT-011, antiPD-1 antibody, enhances ex vivo T-cell responses to autologous dendritic cell/myeloma fusion vaccine. JImmunother. 2011;34(5):409-418.

65. Melero I, et al. Monoclonal antibodies against the 4-1BB T-cell activation molecule eradicate established tumors. Nat Med. 1997;3(6):682-685.

66. Lynch DH. The promise of 4-1BB (CD137)-mediated immunomodulation and the immunotherapy of cancer. Immunol Rev. 2008;222:277-286.

67. Knight DA, et al. Host immunity contributes to the anti-melanoma activity of BRAF inhibitors. J Clin Invest. 2013;123(3):1371-1381.

68. Uno $\mathrm{T}$, et al. Eradication of established tumors in mice by a combination antibody-based therapy. Nat Med. 2006;12(6):693-698.

69. Verbrugge I, et al. Radiotherapy increases the permissiveness of established mammary tumors to rejection by immunomodulatory antibodies. Cancer Res. 2012;72(13):3163-3174.

70. Li SY, Liu Y. Immunotherapy of melanoma with the immune costimulatory monoclonal antibodies targeting CD137. Clin Pharmacol. 2013;5(suppl 1):47-53.

71. Johnston RJ, et al. The immunoreceptor TIGIT regulates antitumor and antiviral CD8(+) T cell effector function. Cancer Cell. 2014;26(6):923-937.

72. Murillo O, et al. Therapeutic antitumor efficacy of anti-CD137 agonistic monoclonal antibody in mouse models of myeloma. Clin Cancer Res. 2008;14(21):6895-6906.

73. von Scheidt B, et al. Combined anti-CD40 and anti-IL-23 monoclonal antibody therapy effectively suppresses tumor growth and metastases. Cancer Res. 2014;74(9):2412-2421. 